\title{
STOCHASTIC REACTIVE SCHEDULING MODEL FOR OPERATING ROOMS CONSIDERING THE MORAL AND HUMAN VIRTUES
}

\author{
GERAMI, F. ${ }^{*}$ - SAIDI-MEHRABAD, M. \\ Department of Industrial Engineering, Iran University of Science and Technology \\ Narmak, Tehran, Iran \\ (phone: +98-21-7322-5000; fax: +98-21-7322-5002) \\ *Corresponding author \\ e-mail: geramifarzad@yahoo.com \\ (phone: +98-31-3651-8673; fax: +98-31-3651-6098) \\ (Received $18^{\text {th }}$ Aug 2016; accepted $15^{\text {th }}$ Nov 2016)
}

\begin{abstract}
This study focuses on the issue of reactive scheduling for the operating room under the uncertain, arrival non-elective patients (emergency or urgent). For this a reactive rescheduling algorithm was proposed and also for steps of algorithm, several stochastic mixed integer programming models were developed. In these models, all duration, such as length of stay in the waiting room, the length of stay in the recovery room, surgery time, operating room preparation time, patient preparation time after anesthesia, patient awake time before transferring to the recovery room and operating room cleaning time are stochastic. The fact that this model is a chance-constraint problem, it looks very complex. In addition to financial goals (overtime, idle time), the important approach in this research is moral and humanitarian objectives such as patient satisfaction, justice, individual freedom, patient rights and medical ethics. In mathematical schedule models, we consider three level of patient's priority $\left(\mathrm{F}_{\mathrm{p}}{ }^{1}, \mathrm{~F}_{\mathrm{p}}{ }^{2}, \mathrm{~F}_{\mathrm{p}}{ }^{3}\right)$. Based on these moral and human dimensions, the decision maker can prioritize patients. Restrictions on parts before (waiting room) and after (PACU) the operation room are also included in the mathematical models. For the evaluation of the proposed model the actual data related to the surgical center of a first-class hospital has been used.
\end{abstract}

Keywords: humanitarian objectives, operating room, surgeries rescheduling, stochastic mixed integer programming, chance-constraint problems

\section{Introduction}

A large share of the costs and revenues of a hospital is related to operating rooms. Therefore, increasing productivity within the operating rooms has a significant impact on the ultimate performance of a hospital. Improved performance in operating rooms also increases economic indicators, rate of services and patients' satisfaction.

Operating room scheduling problem is a special mode of the optimization problem. A lot of researches have been done on the optimization. Hajnal et al. (2007), Kaur (2007) and Tolner (2008) are tree good example. Operating room scheduling problem has some special features that have complicated the problem. Some of these features are:

- ethical and human relationship between the surgeon, patient and nurse;

- human factor (surgeon, patient and nurse) satisfaction;

- patient rights prism;

- the principles of medical ethics; and

-justice. 
For this reason, recently, attention has been given to research in the field of operating room planning and scheduling. Researches related to the Boldy (1976), Blazewich et al. (1983) and Smith et al. (1988) are basic ones in the areas of planning and scheduling of operating rooms. Some writers, like Magerlein and Martin (1978), have given classified literature based on solving methods. But Cardoen et al. (2010) have presented a more comprehensive classification in six areas related to the characteristics (such as performance measures or types of patients) and technical parameters (e.g. solution methods or considering uncertainties) and they have reviewed articles in this field.

Lovejoy and $\mathrm{Li}$ (2002) are thinking of how to increase operating room capacity. They aim at reducing the waiting time and increasing profits and by giving capacity allocation to surgical teams, they have designed the planning and scheduling of operating rooms. Dexter et al. (2003), with the aim of increasing the efficiency of the operating room, have analyzed different scenarios, using event-based simulation. In this model the duration of surgery and also patient arrival are uncertain. Chaabane et al. (2008) believe that operating rooms with appropriation more than $10 \%$ of operating budget, are the most expensive part of a hospital. Their paper introduces two methods for operating room planning and evaluates them.

Belien and Demeulemeester (2007) have focused on the nurses' working pressure of the operating room due to the workload. They have offered a model that integrates the process of scheduling nurses and the operating room. The idea of this model is that, firstly, due to surgery constraints, a Master Surgery Schedule (MSS) is produced. Then, by taking into account the contribution of different types of surgeries, nursing workload distribution is obtained.

Nunes et al. (2009), using a Markov decision process, have modeled elective patients admission control and by using the value iteration algorithm, they have implemented hypothetical examples. Sciomachen et al. (2005) have developed and tested four eventbased simulation models. In their article, sequencing of operations are also analyzed by using the rules of priority. Shamayleh et al. (2012) have considered the issue of operating room capacity planning decisions on the combination of open operating rooms. They have used the integer programming model, to combine the operating room capacity decisions and operating room time allocation decisions. Capacity decisions include determining combination of the number of operating rooms that must be opened and the amount of overtime (to meet demand). It is a certain decision model for elective patients.

Also Baumgart et al. (2007) and Persson and Persson (2010) have used the simulation models as a tool to improve strategic and operational decision making in the delivery of services for the management and planning of surgical procedures in the operating room. Testi et al. (2007) in a certain case where the type of patients is unknown, have developed a three-step approach for the operating room weekly scheduling. This paper proposes an integrated approach for planning surgical operations, to improve efficiency of operating room performance and reduce overtime and patient waiting time. Saremi et al. (2013) have explored a several stages scheduling problem (allocation of surgical services to outpatients). In this model, services time are stochastic.

Meskens et al. (2013) have presented a multi objective model based on the desires and requirements of the surgical team. Their goals are the minimizing the time to do things, minimizing overtime time and maximizing the efficacy of the surgical team and solving it by the genetic algorithm in the real world. Their strategy is the block 
scheduling strategy. The problem is proposed for elective patients under certainty. It also assumes that all the equipment and materials needed are available.

Few researches have been done in reactive scheduling. Most of these researches are in relation to the production scheduling and very few are related to the operating rooms reactive scheduling. However, as will be explained in the next section, there is a clear difference between reactive planning and scheduling in production problem and operating room problem.

Fahmy et al. (2014) in their study have defined the dynamic scheduling. They were divided the dynamic scheduling into three categories: reactive, proactive and predictivereactive. Chaari et al. (2014) have raised four types of scheduling in describing the scheduling problems under uncertainty: proactive, reactive, proactive-reactive and predictive-reactive. Gomes et al. (2015) considered the make to order flexible job shop problem with reentrant process and assembly. They have used a mathematical approach. Farrokhi et al. (2014) have suggested the techniques which can solve reactive scheduling problem in the operating room and then evaluate them for ranking. They have tried to represent and compare and rank the most common techniques by applying the analytic hierarchy process (AHP) and fuzzy technique for order preference by similarity to ideal solution (TOPSIS).

Chung et al. (2012) have presented a reactive scheduling model to minimize makespan of parallel machine problem with job arrival in uncertainty. Cardin et al. (2015) have applied the predictive scheduling and reactive control in manufacturing hybrid control architectures. The aim of Abdesselam et al. (2015) is to formulate a model that solves the reactive flow shop scheduling problem subject to the arrival of new orders. Objective function for makespan minimization and the comprehensive equations for predictive and reactive schedules have been presented with the necessary elaboration.

Nouaouri et al. $(2010,2011)$ have focused on the insertion of unexpected new victim. They have developed a reactive approach algorithm that consists of several integer linear programs. Stuart and Kozan (2012) have presented a model for reactive scheduling in operating room. The problem is modelled as a single machine scheduling problem with sequence dependent processing times and due dates. In Addis et al. (2014a,b) a cardinality constrained robust optimization approach based on Bertsimas and Sim (2004) is proposed. Addis et al. (2015) have considered the problem of selecting a set of patients among a given waiting list of elective patients and have assigned them to a set of available operating room blocks. They have proposed a socalled rolling horizon approach for the patient selection and assignment. They have also proposed a robust formulation of the ILP problem to keep limited the number of disruptions due to uncertain surgery duration. Wang et al. (2015) have focused on the surgery scheduling problem for single surgical suite subject to random emergency surgery. A predictive-reactive scheduling approach is developed to accommodate the influence of surgery's arrival on planned schedule while optimizing the objective of combining efficiency and stability.

Based on the above research papers, the innovations and features of this work in predictive-reactive scheduling can be mentioned as follows, considering human dimensions:

- patient rights' prism (the model is intended for patients' three levels of priority and pays special attention to the rights of vulnerable groups such as children, 
pregnant women, the elderly, psychiatric patients, prisoners, the mentally and physically disabled);

- the principles of medical ethics;

- justice;

- individual freedom (for each scheduling model, a revised approach will be considered, for example, after the initial response, if the surgeon has a special view, again, scheduling is done according to the surgeon s view);

- moral; and

- patient and surgeon (and the others, such as nurses) satisfaction.

Based on these moral and human dimensions, the decision maker can prioritize patients (see in Mathematical models section, Steps $h, j$ ). In mathematical schedule models, we consider three levels of patients' priority $\left(\mathrm{F}_{\mathrm{p}}{ }^{1}, \mathrm{~F}_{\mathrm{p}}{ }^{2}, \mathrm{~F}_{\mathrm{p}}{ }^{3}\right)$ considering the below factors and viewpoints:

- Stochastic surgery time;

- Stochastic other time such as the length of stay in the waiting room, the length of stay in the recovery room, operating room preparation time, patient preparation time after anesthesia, patient awake time before transferring to the recovery room and operating room cleaning time;

- Non-electives arrival (emergency and urgent);

- Separation surgery time from other times (the surgeon is present only during surgery in the operating room);

- Time balancing in the use of operating rooms;

- Balancing on allocation of patients with priority to operating rooms;

- Considering the preference for special patients such as travelers, children, the elderly and people with disabilities;

- Considering the constraints on the availability of equipment needed for surgery; and

- Considering the restrictions on the parts of the before operating room (men's waiting room and women's waiting room separately - due to the specific requirements of hospitals in Iran, the waiting room is intended for men and women separately) and after operating room (PACU).

Some of the previous studies, such as Denton and Gupta (2003) and Atighechian (2012), have partly investigated the different aspects of the operating room scheduling and machine scheduling problem. It is believed that these two issues are completely different. Taken together the reasons stated in previous research papers as well as the direct experience of researchers in this study, the main aspects of differentiation between the operating room scheduling and machine scheduling problem are briefly mentioned. The funds will be classified into four major categories:

1. Problem human dimensions: We face in the operating room scheduling problems with the human aspects such as patient rights' prism, the principles of medical ethics, justice, individual freedom, moral, patient and surgeon satisfaction and human interactions. However, these do not exist in the machine scheduling problems). 
2. Resources: In the operating room scheduling, planning is done according to multiple resources such as operating room, surgeons, technicians and equipment. While, production planning considers only one resource (machine).

3. Type of problem (Solution): In researches related to the production scheduling, the exact solution methods are used. The type of problem that surgeries are affected by special conditions, requires that stochastic solutions be considered (instead of the exact solution). In the analysis conducted by the researchers (in this work), the difference results from the two methods (exact solution and stochastic solution) is quite impressive.

4. Other aspects: There are some other conditions in the operating room scheduling that do not exist in the production planning, such as the absence of the disease before the specified time, lack of commitment by the surgeon who does not come on time, lack of commitment by the surgeon doing overtime and lack of commitment to accountability by the surgeon. While in production, all components or parts are ready at the beginning of the shift, also, the operator must obey all rules such as timely presence, wasted time and overtime.

In the second part of this research, we present the statement of the problem and develop a reactive scheduling algorithm for our problem. The third section presents the stochastic mathematical models. Calculations and numerical results are presented in Computational experiments section. Finally, Conclusions section contains the conclusion and recommendations.

\section{Problem statement}

An issue that is addressed in this study, is a continuation of Gerami's and Saidi's previous researches (the researchers of this study). In Gerami and Saidi (2014a, 2014b) two stochastic scheduling models for surgeries allocation (Integrated Stochastic Surgery Allocation Model (ISSAM)) and also surgeries sequencing (Integrated Stochastic Surgery Sequencing Model (IS $\left.{ }^{3} \mathrm{M}\right)$ ) have been developed. For each issue, a mixed integer programming model is presented. Gerami and Saidi (2014a, 2014b) have brought in their researches that elective surgeries planning and scheduling follow the two steps. The first phase (surgery planning) involves the allocation of elective surgeries to various periods during the planning period, usually weekly, in order to minimize the associated costs. In the second phase (surgery scheduling), sequencing, resource allocation and the surgery start time on a specified date are determined.

It is now the case that non-elective patients (emergency or urgent) enter after the implementation of the basic schedule for elective patients (surgery planning and surgery scheduling). With the approach of making minimal changes on the basic schedule, the proposed models in this paper are for the rescheduling in order to perform surgery on the emergency or urgent patients.

This study focuses on the issue of rescheduling the operating room under uncertainty (length of stay in the waiting room, the length of stay in the recovery room, surgery time, operating room preparation time, patient preparation time after anesthesia, patient awake time before being transferred to the recovery room and operating room cleaning time are stochastic). The important point in this research is that moral and humanitarian objectives (patient satisfaction, justice, individual freedom, patient rights and medical ethics) have been considered, in addition to financial goals (overtime, idle time). 
Restrictions on parts before (waiting room) and after (PACU) the operation room is also included in the model.

It is important that the previous studies have considered only emergency patients. This means that surgery (operation) must be done immediately after the arrival of emergency patients. But in this study, in addition to emergency patients, urgent patients have been included in reschedule process. In other words, there is no need to perform immediate surgery on the patient and the surgery can be done immediately or after a few hours. In this regard, medical standard divides the non-elective patients based on the time allowed for surgery on three levels: 6 hours, 18 hours and 36 hours.

Suppose that a basic schedule for electives in order to perform surgery on date $t$ is existed and implemented. However, during program execution, a non-elective patient enters (emergency or urgent) the hospital. Fig. 1 (proposed algorithm) presents a rescheduling algorithm for operating rooms after entering the emergency or urgent. The algorithm steps are:

a: After emergency or urgent patient entrance, according to some factors such as equipment and facilities, the possible operating rooms are determined.

b: For all possible operating rooms (from a), the operating room and the time of emergency or urgent patient entrance for surgery are calculated (using mathematical model in the next section, Step $b$ ).

c: If the results of Step $b$ is acceptable and feasible, then the algorithm ends.

d: For each possible operating room (from a), the sum of free time is calculated (using mathematical model in the next section, Step $d$ ).

e: The operating room with maximum total free time is selected.

f: For operating room with maximum total free time, the time of emergency or urgent patient entrance for surgery is determined (using mathematical model in the next section, Step $f$ ).

g: If the results of Step $f$ affect the other operating rooms schedule, then the Step $j$ is activated.

h: The remaining patients in the operating room with maximum total free time is reschedule (using mathematical model in the next section, Step $h$ ).

i: If the results of Step $h$ is acceptable and feasible, then the algorithm ends.

j: The remaining patients in all operating room, are reschedule (using mathematical model in the next section, Step $j$ ).

\section{Mathematical models}

\section{Symbols}

The following symbols are used in this paper:

\section{Indices}

$p$ : patient (surgery)

pe: non-elective (emergency or urgent) patient

$o$ : operating room

$s$ : surgeon 


\section{Parameters}

$N$ : the number of patients demanding surgery

$P$ : number of patients

0 : number of operating rooms

$S$ : number of surgeons

$T_{\circ}$ : normal duration of the operating room $o$ (minute)

$\mathrm{Ta}_{\mathrm{o}}$ : allowed overtime of the operating room ${ }^{\circ}$ (minute)

$d s_{p}$ : operating room preparation time of patient $p$ (including operating room preparation time, patient preparation time, anesthesia time)

$f_{d s_{p}}(x)$ : probability distribution function of $d s_{p}$

$d a_{p}$ : surgery time of patient $p$

$f_{d a_{p}}(x)$ : probability distribution function of $d a_{p}$

$d p o_{p}$ : the duration of the process of care after anesthesia for patient $p$ which is done in the operating room

$f_{d p o_{p}}(x)$ : probability distribution function of $d p o_{p}$

$d c_{p}$ : operating room cleaning time of patient $p$

$f_{d c_{p}}(x)$ : probability distribution function of $d c_{p}$

$d p_{p}$ : duration of post anesthesia until stabilization of patient $p$

$f_{d p_{p}}(x)$ : probability distribution function of $d p_{p}$

$d w_{p}$ : length of stay in the waiting room of patient $p$

$f_{d w_{p}}(x)$ : probability distribution function of $d w_{p}$

$D_{p}: \quad D_{p}=d s_{p}+d a_{p}+d c_{p}$

$f_{D_{p}}(x)$ : probability distribution function of $D_{p}$

$d p_{p e}$ : emergency or urgent patient ( $p e$ )'s preparation time before entering the operating room

$f_{d p_{p e}}(x)$ : probability distribution function of $d p_{p e}$

$d s_{p e}$ : preparation time of non-elective patient pe in operating room

$f_{d s_{p e}}(x)$ : probability distribution function of $d s_{p e}$

$\left[L_{S}, U_{s}\right]$ : presence time of surgeon $s$

OT: opening time of department of Surgery on day $t$

$B W m$ : the number of beds available in men's waiting room

$B W w$ : the number of beds available in women's waiting room

$B P$ : the number of beds available in PACU

$\operatorname{LOFLT}_{\circ}$ : the latest time of completion the latest surgery in the operating room $o$

LOFLTE $_{\circ}$ : the latest time of completion the non-elective surgery in the operating room $o$

$S F L T_{g}$ : the latest time of finishing the surgeon time

$F T_{o}$ : total of free time for operating room $o$

$E F T_{p}$ : the earliest time of completion the surgery $p$

$L F T_{p}$ : the latest time of completion the surgery $p$ 


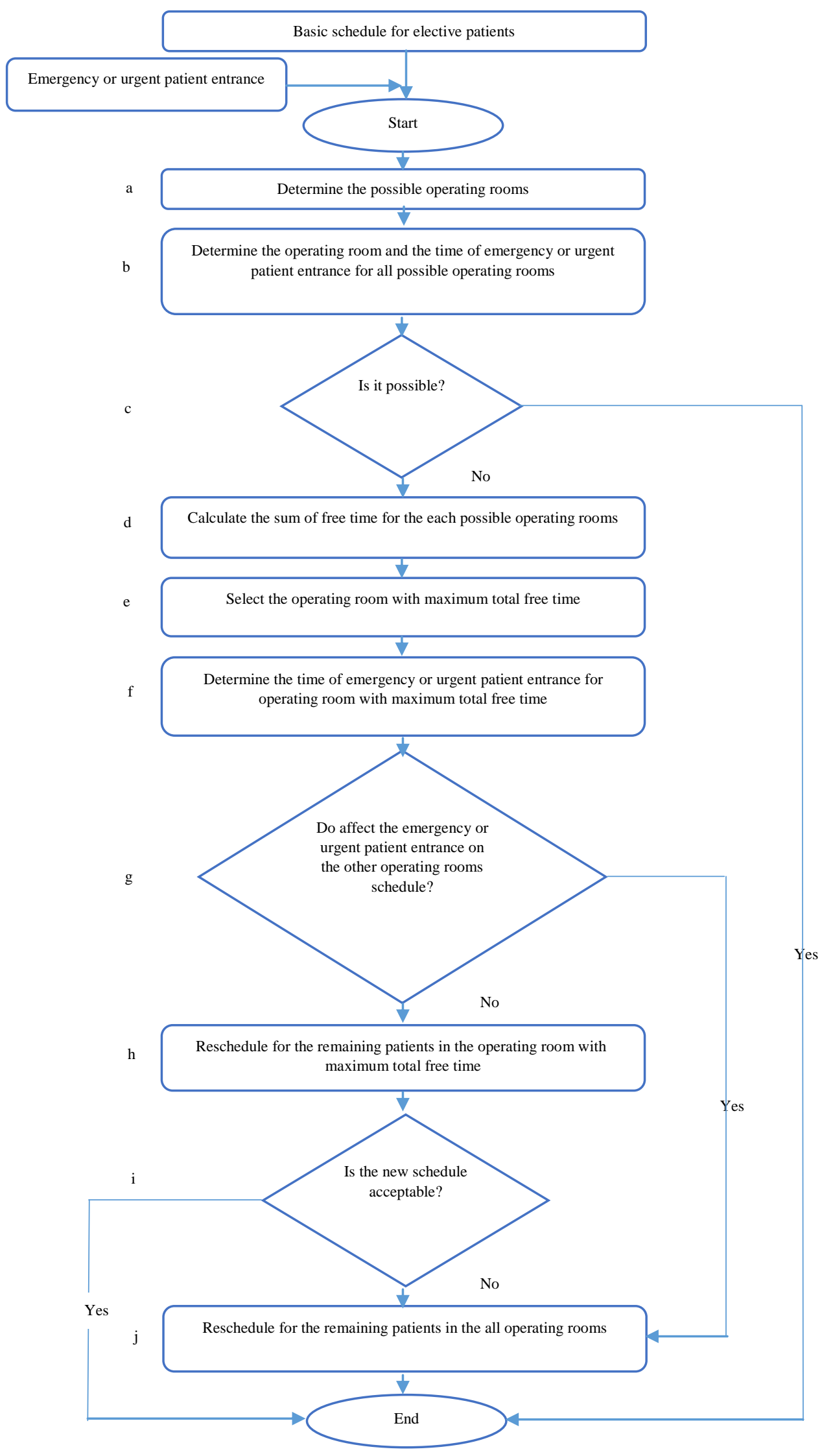

Figure 1. Rescheduling algorithm (reactive scheduling models) for operating rooms after arriving emergency or urgent patients

APPLIED ECOLOGY AND ENVIRONMENTAL RESEARCH 15(3): 563-592.

http://www.aloki.hu • ISSN 15891623 (Print) • ISSN 17850037 (Online)

DOI: http://dx.doi.org/10.15666/aeer/1503_563592

(C) 2017, ALÖKI Kft., Budapest, Hungary 
$E L T_{p e}:$ the latest arrival time of non-elective patients pe to operating room (deadline)

CT: surgery forums closing time

$t a_{p e}:$ the arrival time of non-elective patients pe to hospital

$A T_{\circ}$ : availability time of operating room $o$

$T H$ : time horizon $\left(\mathrm{OT}+T_{\circ}+T a_{\circ}\right)$

$M, M_{1}, M_{2}, M_{3}$ : a large number

\section{Decision variables}

$x_{p, o}: 1$ if patient $p$ is allocated to operating room $o, 0$ otherwise

$\xi_{p, p}:$ if the patient $p$ is entering the waiting room before the patient $p^{\prime}, 0$ otherwise

$\alpha_{p, b m}: 1$ if the patient $p$ is allocated to bed $b m$ in men's waiting room

$\beta_{p, b w}: 1$ if the patient $p$ is allocated to bed $b w$ in men's waiting room

$3_{p, p}: 1$ if the patient $p$ is entering the operating room before the patient $p^{\prime}, 0$ otherwise

$v_{p, p}: 1$ if the patient $p$ is entering the PACU before the patient $p^{\prime}, 0$ otherwise

$\delta_{p, b p}: 1$ if the patient $p$ is allocated to bed $b p$ in PACU

$\pi_{p, s}: 1$ when surgeon $s$ is allocated to patient $p$ (to perform operation), 0 otherwise

$\varepsilon_{p, o}: 1$ when the equipment needed for surgical procedure $p$ is available in operating room $o, 0$ otherwise

$F_{p}^{1}: 1$ when the patient $p$ has the lowest priority for surgery on day t, 0 otherwise

$F_{p}^{2}: 1$ when the patient $p$ has the medium priority for surgery on day t, 0 otherwise

$F_{p}^{3}: 1$ when the patient $p$ has the highest priority for surgery on day t, 0 otherwise

$\lambda_{p}: 1$ when the patient $p$ is a man, 0 otherwise

$x_{p \varepsilon_{0}, t}: 1$ if non-elective patient pe is allocated to operating room $o$ on time $\mathrm{t}, 0$ otherwise

$\pi_{\mathrm{pe}, \mathrm{s}}: 1$ when surgeon $s$ is allocated to non-elective patient pe (to perform operation), 0 otherwise

\section{Positive real variable}

$t w_{p}:$ start time in waiting room for patient $p$

to ${ }_{p}$ : start time in operating room for patient $p$

to $o_{p e}$ : start time in operating room for non-elective patient $p e$

$t p_{p}$ : start time in PACU for patient $p$

\section{Mathematical models of operating rooms rescheduling (reactive scheduling) after entering non-elective patient (emergency or urgent)}

In this case, the operating room has been considered with the part of before operating room including men's waiting room and women's waiting room (because of the special regulatory requirements of hospitals in Iran) and also the part of the after surgery including PACU. Fig. 2 schematically illustrates this show. Figure 3 presents a conceptual model used in this problem. 


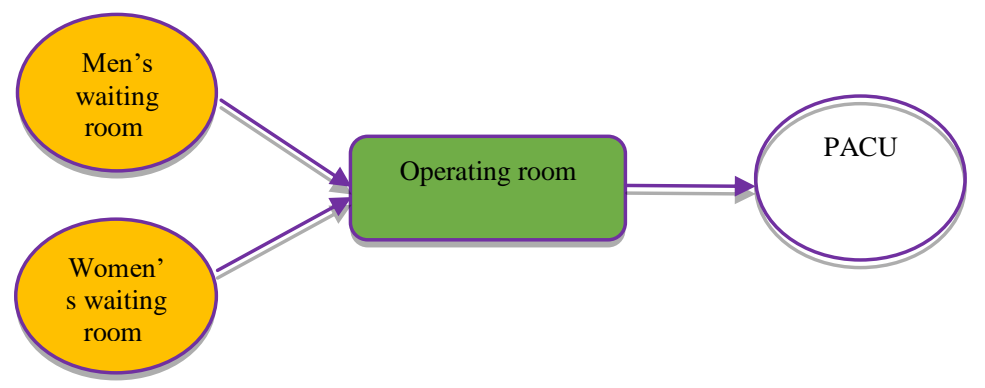

Figure 2. The part of the before (men's waiting room and women's waiting room) and after operating room (PACU)

Due to the rescheduling algorithm (Fig. 1), for the Steps $b, d, f, h$ and $j$ the following mathematical models have been developed.

Mathematical model to determine the operating room and the time of emergency or urgent patient entrance for all possible operating rooms (Step b)

This model aims to minimize the emergency or urgent patient entrance time the operating room considering the latest completion time of the last surgery in the operating room (Eq. 1).

$$
\operatorname{Min} x_{0}=\sum_{o=1}^{O} \sum_{t=L O F L T_{0}}^{T H} \text { to }{ }_{p e^{*}} x_{p \theta_{0}, t}
$$

Constraints of this model are formulated as follows:

$$
\begin{aligned}
& \sum_{o=1}^{O} \sum_{t=L O F L T_{0}}^{T H} x_{p \theta_{i}, t} \leq 1 \\
& E L T_{p e} \cdot \sum_{o=1}^{O} \sum_{t=L O F L T_{0}}^{T H} x_{p \theta_{i}, t}-t o_{p e} \geq \mathrm{M}\left(\sum_{o=1}^{O} \sum_{t=L O F L T_{0}}^{T H} x_{p \theta_{i}, t}-1\right) \text { (Eq. 3) } \\
& \text { to } o_{p e}-\left(t a_{p e}+d p_{p e}\right) \geq \mathrm{M}\left(\sum_{o=1}^{O} \sum_{t=L O F L T_{0}}^{T H} x_{p \theta_{i}, t}-1\right) \\
& \text { to } o_{p e}-\pi_{\mathrm{pe}, \mathbb{s}^{*}} S F L T_{\mathbb{s}^{*}} \sum_{t=L O F L T_{0}}^{T H} x_{p \theta_{2}, t, t} \geq \mathrm{M}\left(\sum_{t=L O F L T_{0}}^{T H} x_{p \theta_{i}, t, t}-1\right) \forall \mathrm{o} \in[1, \ldots, 0](\mathrm{Eq} .5) \\
& \text { to } o_{p e}=\sum_{o=1}^{O} \sum_{t=L O F L T_{0}}^{T H} \text { t. } x_{p \theta_{i}, t}-\mathrm{M}\left(\sum_{o=1}^{O} \sum_{t=L O F L T_{0}}^{T H} x_{p e_{0}, t}-1\right) \\
& x_{p \theta_{0}, t} \in\{0,1\} \forall \mathrm{o} \in[1, \ldots, \mathrm{O}], \forall \mathrm{t} \in\left[L O F L T_{\mathrm{o}}, \ldots, \mathrm{TH}\right]
\end{aligned}
$$

The constraint (2) specifies that any operation can be assigned at most once. The constraint (3) checks the last deadline $\left(E L T_{p e}\right)$ for enter emergency or urgent patient to the operating room $\left(t_{p e}\right)$. Formula (4) refers to the emergency or urgent patient entrance time the operating room must be after the time of preparation (before entering the operating room). Constraint (5) checks the start time of surgery considering the presence time of surgeon. The constraint (6) determines the emergency or urgent patient entrance time the operating room. The constraint (7) ensure the integrality of the variable. 


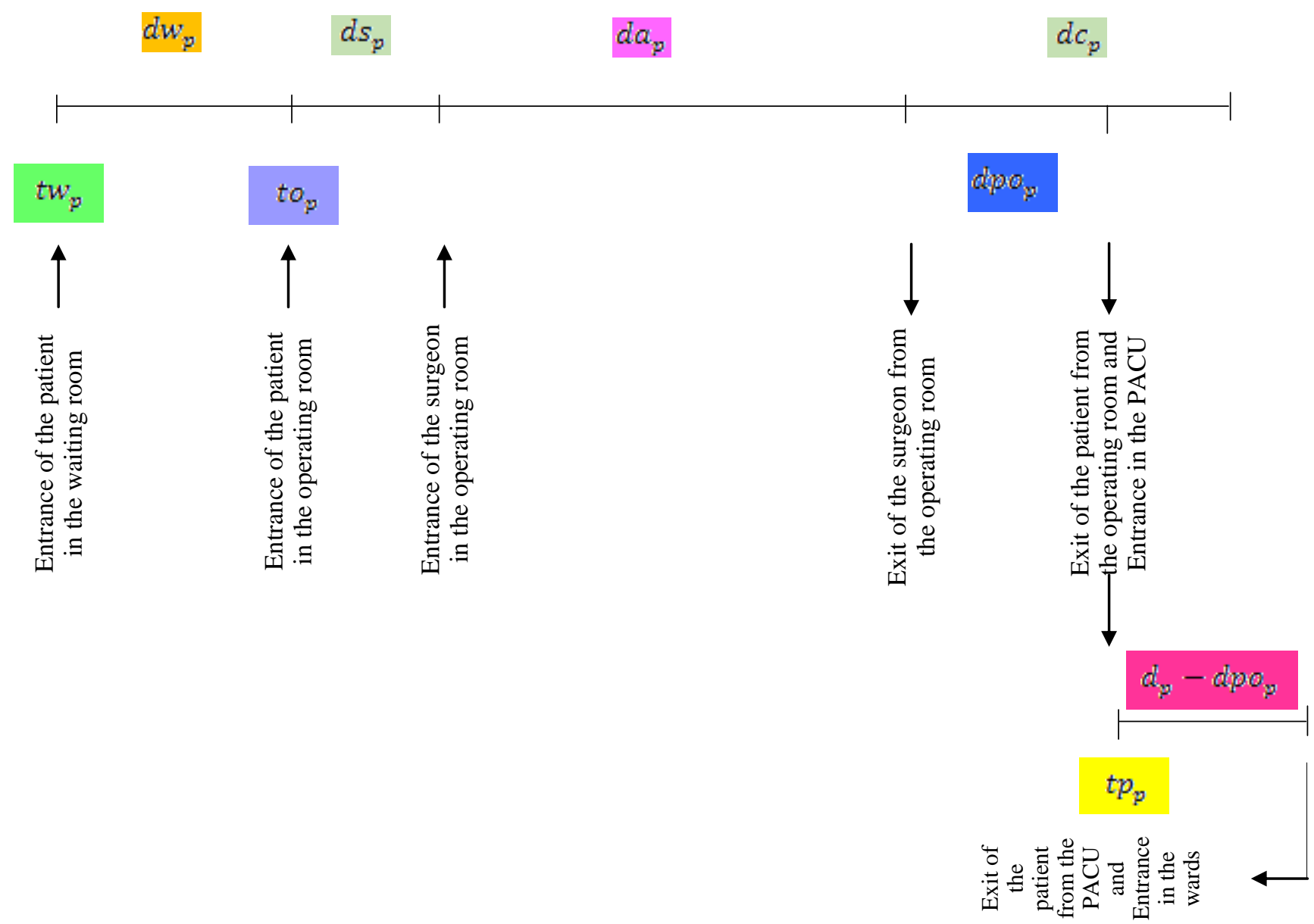

Figure 3. Conceptual model (the entrance and exit of the patient and the surgeon)

DOI: http://dx.doi.org/10.15666/aeer/1503 563592

() 2017, ALÖKI Kft., Budapest, Hungary 
Mathematical model to calculate the sum of free time for the each possible operating rooms (Step d)

This model calculates the sum of free time for the each possible operating rooms by adding free time at the end of the operating room and the floating time between surgeries (Eq. 8), as shown in Fig 4.

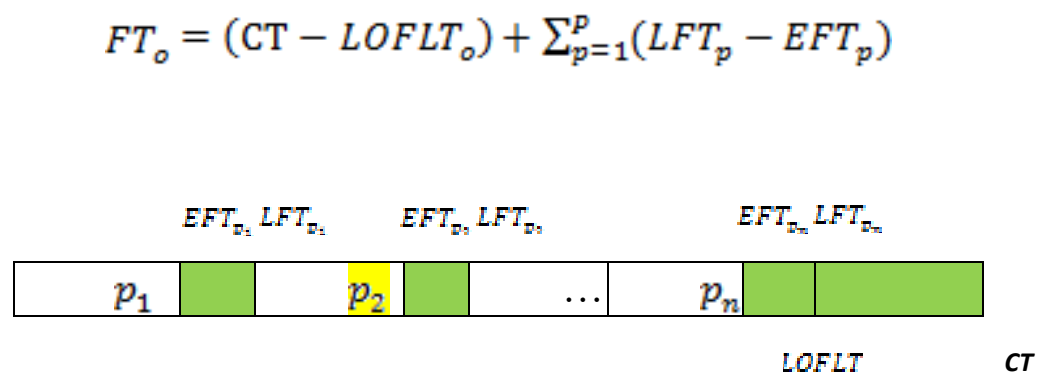

Figure 4. Sum of free time for an operating room

Mathematical model to determine the time of emergency or urgent patient entrance for operating room with maximum total free time (Step $f$ )

This model aims to maximize the emergency or urgent patient entrance time the operating room (to have less impact on the basic schedule and having minimal changes in other patients plan) (Eq. 9).

$$
\operatorname{Max} x_{0}=\sum_{t a_{p e}}^{T H}\left(t o_{p e}-t a_{p \theta}\right) \cdot x_{p \theta_{0}, t}
$$

Constraints of this model are formulated as follows:

$$
\begin{aligned}
& \sum_{t \alpha_{p \theta}}^{T H} x_{p \theta_{0}, t} \leq 1 \\
& x_{p \theta_{i}, t}=1 \quad \forall \mathrm{t} \in\left[t a_{p e}, \ldots, \mathrm{TH}\right] \\
& E L T_{p e^{*}} \sum_{t a_{p e}}^{T H} x_{p \theta_{i}, t}-t o_{p e} \geq 0 \\
& \text { to } o_{p e}-\left(t a_{p e}+d p_{p e}\right) \geq 0 \\
& \text { to } o_{p e}+d s_{p e} \geq \pi_{p e_{s} \varepsilon^{*}} L_{s} \forall \mathrm{s} \in[1, \ldots, \mathrm{S}] \\
& t_{p e} \geq \mathrm{AT}_{\sigma^{\circ}}, x_{p \theta_{i}, t} \quad \forall \mathrm{t} \in\left[t a_{p e}, \ldots, \mathrm{TH}\right] \\
& \text { to } o_{p e}=\sum_{t a_{p e}}^{T H} t_{t} x_{p \theta_{0}, t} \\
& x_{p \theta_{i}, t} \in\{0,1\} \quad \forall \mathrm{t} \in\left[t a_{p_{e}}, \ldots, \mathrm{TH}\right]
\end{aligned}
$$

Constraints (10) checks that emergency operation can be assigned at most once. Formula (11) refers to the fact that patients with priority should be to allocate. 
Constraints (12) consider the last deadline $\left(E L T_{p e}\right)$ for enter emergency or urgent patient to the operating room. Constraint (13) ensures that the emergency or urgent patient entrance time the operating room must be after the time of preparation (before entering the operating room). Also restriction (14) ensures the start time of surgery considering the presence time of surgeon. The constraint (15) checks the time of operating room availability. The constraint (16) calculates the emergency or urgent patient entrance time the operating room. The constraint (17) ensure the integrality of the variable $x_{p \theta_{i, 0}, t}$.

Mathematical model to reschedule for the remaining patients in the operating room with maximum total free time (Step $h$ )

This model aims to minimize the sum of start time in PACU (Eq. 18). According to patients' priority, $M_{1}, M_{2}$ and $M_{3}$ are different. So this model makes the most effort to minimize the exit time of patients with a higher priority.

$\operatorname{Min} x_{o}=M_{1} \cdot \sum_{p=1}^{N} F_{p}^{1} \cdot t p_{p}+M_{2} \cdot \sum_{p=1}^{N} F_{p}^{2} \cdot t p_{p}+M_{3} \cdot \sum_{p=1}^{N} F_{p}^{3} \cdot t p_{p}$

In this mathematical schedule models, we consider three level of patient's priority $\left(F_{p}^{1}, F_{p}^{2}, F_{p}^{3}\right)$. Based on these moral and human dimensions, decision maker can prioritize patients. Constraints of this model are formulated as follows:

$$
\begin{aligned}
& t w_{p} \geq L O F L T E_{o} \quad \forall p \in[1, \ldots, N] \\
& \text { to }_{p} \geq \operatorname{LOFLTE} \quad \forall p \in[1, \ldots, N] \\
& t p_{p} \geq \operatorname{LOFLTE} \quad \forall p \in[1, \ldots, N] \\
& \left(t o_{p}+d s_{p}\right)-\left(t o_{p}+d s_{p}+d a_{p}\right) \geq M\left(\pi_{p, s}+\pi_{p, s}+z_{p, p}-3\right) \\
& \forall p, \hat{p} \in[1, \ldots, N], p \neq \hat{p}, \forall s \in[1, \ldots, S] \\
& \text { to } o_{p}+d s_{p} \geq \pi_{p, s} . L_{s} \forall p \in[1, \ldots, N], \forall s \in[1, \ldots, S] \\
& t o_{\hat{p}}-\left(t o_{p}+D_{p}+d p o_{p}\right) \geq M\left(x_{p, o}+x_{\hat{p}, 0}+3_{p, p}-3\right) \forall p, \hat{p} \in[1, \ldots, N], p \neq \hat{p} \\
& t p_{p}-\left(t p_{p}+d p_{p}-d p o_{p}\right) \geq M\left(\delta_{p, b p}+\delta_{p, b p}+v_{p, p}-3\right) \\
& \forall p, \hat{p} \in[1, \ldots, N], p \neq \hat{p}, \forall b p \in[1, \ldots, B P] \\
& 3_{p, p}+3_{p, p}=1 \forall p, \hat{p} \in[1, \ldots, N], p>\hat{p} \\
& \text { to } o_{\hat{p}}-\text { to }_{p} \geq M\left(3_{p, p}-1\right) \quad \forall p, \hat{p} \in[1, \ldots, N], p \neq \hat{p} \\
& v_{p, p}+v_{\hat{p}, p}=1 \forall p, \hat{p} \in[1, \ldots, N], p>\hat{p}
\end{aligned}
$$




$$
\begin{aligned}
& t p_{\hat{p}}-t p_{p} \geq M\left(v_{p, p}-1\right) \quad \forall p, \hat{p} \in[1, \ldots, N], p \neq \hat{p} \\
& t p_{p}=t o_{p}+d s_{p}+d a_{p}+d p o_{p} \forall p \in[1, \ldots, N] \\
& d p o_{p} \leq d p_{p} \forall p \in[1, \ldots, N] \\
& F_{p}^{1}+F_{p}^{2}+F_{p}^{3}=1 \forall p=1,2, \ldots, P \\
& \xi_{p, p^{\prime}}=1 \quad \forall p, p \in[1, \ldots, \hat{N}], p \neq \hat{p}, F_{p}^{3}=1, F_{p^{r}}^{3}=0 \\
& 3_{p, p}=1 \quad \forall p, p \in[1, \ldots, N], p \neq \hat{p}, F_{p}^{3}=1, F_{p^{r}}^{3}=0 \\
& \xi_{p, \hat{p}}=1 \quad \forall p, p \in[1, \ldots, N], p \neq \hat{p}, F_{p}^{2}=1, F_{p^{t}}^{1}=1 \\
& 3_{p, p}=1 \quad \forall p, p \in[1, \ldots, \hat{N}], p \neq \hat{p}, F_{p}^{2}=1, F_{p^{t}}^{1}=1 \\
& t w_{p}-\left(t w_{p}+d w_{p}\right) \geq M\left(\alpha_{p, b m}+\alpha_{\hat{p}, b m}+\xi_{p, p}-3\right) \\
& \forall p, \hat{p} \in[1, \ldots, N], \forall b m \in[1, \ldots, B W m], \lambda_{p} \lambda_{p}=1 \\
& t w_{p}-\left(t w_{p}+d w_{p}\right) \geq M\left(\beta_{p, b w}+\beta_{p, b w}+\xi_{p, p}-3\right) \\
& \forall p, \hat{p} \in[1, \ldots, N], \forall b w \in[1, \ldots, B W w],\left(1-\lambda_{p}\right)\left(1-\lambda_{p}\right)=1 \\
& \xi_{p, p}+\xi_{p, p}=1 \quad \forall p, \hat{p} \in[1, \ldots, N], p>\hat{p} \\
& t w_{\hat{p}}-t w_{p} \geq M\left(\xi_{p, p}-1\right) \forall p, \hat{p} \in[1, \ldots, N], p \neq \hat{p} \\
& \text { to } o_{p}=t w_{p}+d w_{p} \forall p \in[1, \ldots, N] \\
& \sum_{b m=1}^{B W m} \alpha_{p, b m}=1 \forall p \in[1, \ldots, N], \lambda_{p}=1 \\
& \sum_{b w=1}^{B W w} \beta_{p, b w}=1 \forall p \in[1, \ldots, N], \lambda_{p}=0 \\
& \sum_{b p=1}^{B P} \delta_{p, b p}=1 \quad \forall p \in[1, \ldots, N]
\end{aligned}
$$

$$
\sum_{p \in A_{1}} \sum_{b m=1}^{B W m} \alpha_{p, b m} \leq B W m \quad A_{1}=\left\{p \mid O T \leq t w_{p} \leq O T+\overline{d w}\right\}, \lambda_{p}=1
$$

$$
\sum_{p \in A_{2}} \sum_{b m=1}^{B W m} \alpha_{p, b m} \leq B W m \quad A_{2}=\left\{p \mid O T+\overline{d w} \leq t w_{p} \leq O T+2 \overline{d w}\right\}, \lambda_{p}=1
$$

$\sum_{p \in A_{i}} \sum_{b m=1}^{B W m} \alpha_{p, b m} \leq B W m \quad A_{i}=\left\{p \mid O T+2 \overline{d w} \leq t w_{p} \leq O T+i \overline{d w}\right\}, \lambda_{p}=1$ 


$$
\sum_{p \in B_{1}} \sum_{b m=1}^{B W m} \beta_{p, b m} \leq B W W \quad B_{1}=\left\{p \mid O T \leq t w_{p} \leq O T+\overline{d w}\right\}, \lambda_{p}=0
$$

$\sum_{p \in B_{2}} \sum_{b m=1}^{B W m} \beta_{p, b m} \leq B W W \quad B_{2}=\left\{p \mid O T+\overline{d w} \leq t w_{p} \leq O T+2 \overline{d w}\right\}, \lambda_{p}=0$

$$
\sum_{p \in B_{j}} \sum_{b m=1}^{B W m} \beta_{p, b m} \leq B W W \quad B_{j}=\left\{p \mid O T+2 \overline{d w} \leq t w_{p} \leq O T+j \overline{d w}\right\}, \lambda_{p}=0
$$

$$
\begin{gathered}
\sum_{p \in C_{1}} \sum_{b p=1}^{B P} \delta_{p, b p} \leq B P \quad C_{1}=\left\{p \mid O T P \leq t p_{p} \leq O T P+\overline{d p}\right\} \\
\sum_{p \in C_{2}} \sum_{b p=1}^{B P} \delta_{p, b p} \leq B P \quad C_{2}=\left\{p \mid O T P+\overline{d p} \leq t p_{p} \leq O T P+2 \overline{d p}\right\} \\
\sum_{p \in C_{k}} \sum_{b p=1}^{B P} \delta_{p, b p} \leq B P \quad C_{k}=\left\{p \mid O T P+2 \overline{d p} \leq t p_{p} \leq O T P+k \overline{d p}\right\} \text { (Eq. 53) } \\
d p o_{p} \geq 0 \forall p \in[1, \ldots, N] \\
x_{p, o} \in\{0,1\} \forall p \in[1, \ldots, N] \\
\delta_{p, b p} \in\{0,1\} \forall b p \in[1, \ldots, B P], \forall p \in[1, \ldots, N] \\
\alpha_{p, b m} \in\{0,1\} \forall b m \in[1, \ldots, B W m], \forall p \in[1, \ldots, N] \\
\beta_{p, b w} \in\{0,1\} \forall b w \in[1, \ldots, B W w], \forall p \in[1, \ldots, N] \\
3_{p, p} \in\{0,1\} \forall p, \hat{p} \in[1, \ldots, N], p \neq \hat{p} \\
\xi_{p, p} \in\{0,1\} \forall p, \hat{p} \in[1, \ldots, N], p \neq \dot{p} \\
v_{p, p} \in\{0,1\} \forall p, \hat{p} \in \in[1, \ldots, N], p \neq \dot{p} \\
\pi_{p, s} \in\{0,1\} \forall s=1,2, \ldots, S, \forall p=1,2, \ldots, P \\
\lambda_{p} \in\{0,1\} \forall p \in[1, \ldots, N] \\
F_{p}^{1} \in\{0,1\} \quad \forall p=1,2, \ldots, P \\
F_{p}^{2} \in\{0,1\} \quad \forall p=1,2, \ldots, P \\
F_{p}^{3} \in\{0,1\} \quad \forall p=1,2, \ldots, P
\end{gathered}
$$

The constraint (19), (20) and (21) ensures that entrance time of remaining patients in waiting room, operating room and PACU must be after the latest surgery completion time of emergency or urgent patient in the operating room. The constraint (22) ensures 
that the overlap does not occur for each two patients were allocated to a surgeon. The constraint (23) checks the start time of surgery considering the presence time of surgeon. Formula (24) refers to the lack of overlap between the two cases which have been allocated to an operating room. Constraint (25) ensures that the overlap does not occur for each two patients who have been allocated to a bed in recovery room. The constraints (26) and (27) check the transposition in patient entrance to operating room. Also formulas (28) and (29) check the transposition in patient entrance to recovery room. Constraint (30) checks that for each patient the recovery phase begins after completion of the surgery and also awaking the patient in the operating room. Formula (31) ensures that the waking the patient time in operating room is shorter than the stabilization time of the patient's condition in recovery. Constraints (32) to (36) ensure the patient's priority to entrance to waiting room and operating room. Constraint (37)/ (38) ensures that the overlap does not occur for each two men/ women (patients) who have been allocated to a bed in waiting room. Constraints (39) and (40) check the transposition in patient entrance to waiting room. Constraint (41) checks that for each patient the surgery phase begins after the length of stay in the waiting room. Constraints (42) and (43) are preventing from allocating more than once a bed to a patient in waiting rooms for men and women. Restriction (44) considers that a bed in recovery can be allocated to a patient only once. Constraints (45) to (50) check the lack of overlap between the two patients who have been allocated to a bed in waiting room (for men and women) at various time intervals. Also Constraints (51) to (53) check the lack of overlap between the two patients who have been allocated to a bed in PACU at various time intervals. Restriction (54) checks the duration of the process of care after anesthesia for patient $p$ which is done in the operating room to be positive. Constraints (55) to (66) ensure the integrality of the variables.

Mathematical model to reschedule for the remaining patients in the all operating rooms (Step j)

This model is similar to the previous model. The previous model focuses on the operating room with maximum total free time and this model will reschedule for all operating rooms. So the objective and constraints are considered for all operating rooms.

$\operatorname{Min} x_{o}=M_{1} \cdot \sum_{p=1}^{N} F_{p}^{1} \cdot t p_{p}+M_{2} \cdot \sum_{p=1}^{N} F_{p}^{2} \cdot t p_{p}+M_{3} \cdot \sum_{p=1}^{N} F_{p}^{3} \cdot t p_{p}$

Constraints of this model are formulated as follows:

$$
\begin{aligned}
& t w_{p}, x_{p, o}-L O F L T E_{o} \geq M\left(x_{p, o}-1\right) \quad \forall p \in[1, \ldots, N], \forall o \in[1, \ldots, 0] \quad \text { (Eq. 68) } \\
& \text { to }_{p}, x_{p, o}-\text { LOFLTE }_{o} \geq M\left(x_{p, o}-1\right) \quad \forall p \in[1, \ldots, N], \forall \circ \in[1, \ldots, O] \quad \text { (Eq. 69) } \\
& t p_{p}, x_{p, o}-L O F L T E_{o} \geq M\left(x_{p, o}-1\right) \quad \forall p \in[1, \ldots, N], \forall o \in[1, \ldots, 0] \quad \text { (Eq. 70) } \\
& \left(t o_{p}+d s_{p}\right)-\left(t o_{p}+d s_{p}+d a_{p}\right) \geq M\left(\pi_{p, s}+\pi_{p, s}+z_{p, p}-3\right)
\end{aligned}
$$

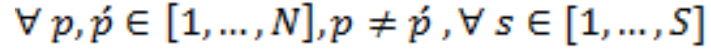




$$
\begin{aligned}
& \text { to } o_{p}+d s_{p} \geq \pi_{p, s} . L_{s} \quad \forall p \in[1, \ldots, N], \forall s \in[1, \ldots, S] \\
& t o_{\hat{p}}-\left(\text { to }_{p}+D_{p}+d p o_{p}\right) \geq M\left(x_{p, o}+x_{p_{i} \circ}+z_{p, p}-3\right) \forall p, \hat{p} \in[1, \ldots, N], p \neq \hat{p} \\
& t p_{p}-\left(t p_{p}+d p_{p}-d p o_{p}\right) \geq M\left(\delta_{p, b p}+\delta_{p, b p}+v_{p, p}-3\right) \\
& p, \hat{p} \in[1, \ldots, N], p \neq \hat{p}, \forall b p \in[1, \ldots, B P] \\
& 3_{p, p}+3_{p, p}=1 \quad \forall p, \hat{p} \in[1, \ldots, N], p>\hat{p} \\
& \text { to } o_{p}-\text { to }_{p} \geq M\left(3_{p, p}-1\right) \forall p, \hat{p} \in[1, \ldots, N], p \neq \hat{p} \\
& v_{p, \hat{p}}+v_{\hat{p}, p}=1 \forall p, \hat{p} \in[1, \ldots, N], p>\hat{p} \\
& t p_{\hat{p}}-t p_{p} \geq M\left(v_{p, p}-1\right) \quad \forall p, \hat{p} \in[1, \ldots, N], p \neq \hat{p} \\
& t p_{p}=t o_{p}+d s_{p}+d a_{p}+d p o_{p} \quad \forall p \in[1, \ldots, N] \\
& d p o_{p} \leq d p_{p} \quad \forall p \in[1, \ldots, N] \\
& F_{p}^{1}+F_{p}^{2}+F_{p}^{3}=1 \quad \forall p=1,2, \ldots, P \\
& \xi_{p, p}=1 \quad \forall p, p \in[1, \ldots, N], p \neq \hat{p}, F_{p}^{3}=1, F_{p^{t}}^{3}=0 \\
& 3_{p, p^{f}}=1 \quad \forall p, p \in[1, \ldots, \hat{N}], p \neq \hat{p}, F_{p}^{3}=1, F_{p^{r}}^{3}=0 \\
& \xi_{p, \hat{p}}=1 \quad \forall p, p \in[1, \ldots, \hat{N}], p \neq \hat{p}, F_{p}^{2}=1, F_{p^{\prime}}^{1}=1 \\
& 3_{p, p}=1 \quad \forall p, p \in[1, \ldots, \hat{N}], p \neq \hat{p}, F_{p}^{2}=1, F_{p^{\prime}}^{1}=1 \\
& t w_{p}-\left(t w_{p}+d w_{p}\right) \geq M\left(\alpha_{p, b m}+\alpha_{p, b m}+\xi_{p, p}-3\right) \\
& \forall p, \hat{p} \in[1, \ldots, N], \forall b m \in[1, \ldots, B W m], \lambda_{p} \lambda_{p}=1 \\
& t w_{p}-\left(t w_{p}+d w_{p}\right) \geq M\left(\beta_{p, b w}+\beta_{p, b w}+\xi_{p, p}-3\right) \\
& \forall p, \hat{p} \in[1, \ldots, N], \forall b w \in[1, \ldots, B W w],\left(1-\lambda_{p}\right)\left(1-\lambda_{p}\right)=1 \\
& \xi_{p, p}+\xi_{p, p}=1 \quad \forall p, \hat{p} \in[1, \ldots, N], p>\hat{p} \\
& t w_{\hat{p}}-t w_{p} \geq M\left(\xi_{p, p}-1\right) \forall p, \hat{p} \in[1, \ldots, N], p \neq \hat{p} \\
& \text { to } o_{p}=t w_{p}+d w_{p} \quad \forall p \in[1, \ldots, N] \\
& \sum_{b m=1}^{B W m} \alpha_{p, b m}=1 \quad \forall p \in[1, \ldots, N], \lambda_{p}=1
\end{aligned}
$$




$$
\begin{gathered}
\sum_{b w=1}^{B W w} \beta_{p, b w}=1 \forall p \in[1, \ldots, N], \lambda_{p}=0 \\
\sum_{b p=1}^{B P} \delta_{p, b p}=1 \quad \forall p \in[1, \ldots, N]
\end{gathered}
$$

$\sum_{p \in A_{1}} \sum_{b m=1}^{B W m} \alpha_{p, b m} \leq B W m A_{1}=\left\{p \mid O T \leq t w_{p} \leq O T+\overline{d w}\right\}, \lambda_{p}=1$

$\sum_{p \in A_{2}} \sum_{b m=1}^{B W m} \alpha_{p, b m} \leq B W m A_{2}=\left\{p \mid O T+\overline{d w} \leq t w_{p} \leq O T+2 \overline{d w}\right\}, \lambda_{p}=1$ (Eq.95)

$\sum_{p \in A_{i}} \sum_{b m=1}^{B W m} \alpha_{p, b m} \leq B W m A_{i}=\left\{p \mid O T+2 \overline{d w} \leq t w_{p} \leq O T+i \overline{d w}\right\}, \lambda_{p}=1$ (Eq.96)

$\sum_{p \in B_{1}} \sum_{b m=1}^{B W m} \beta_{p, b m} \leq B W W B_{1}=\left\{p \mid O T \leq t w_{p} \leq O T+\overline{d w}\right\}, \lambda_{p}=0$

$\sum_{p \in B_{2}} \sum_{b m=1}^{B W m} \beta_{p, b m} \leq B W W B_{2}=\left\{p \mid O T+\overline{d w} \leq t w_{p} \leq O T+2 \overline{d w}\right\}, \lambda_{p}=0$ (Eq.98)

$\sum_{p \in B_{j}} \sum_{b m=1}^{B W m} \beta_{p, b m} \leq B W W B_{j}=\left\{p \mid O T+2 \overline{d w} \leq t w_{p} \leq O T+j \overline{d w}\right\}, \lambda_{p}=0$ (Eq.99)

$\sum_{p \in C_{1}} \sum_{b p=1}^{B P} \delta_{p, b p} \leq B P \quad C_{1}=\left\{p \mid O T P \leq t p_{p} \leq O T P+\overline{d p}\right\}$

$\sum_{p \in C_{2}} \sum_{b p=1}^{B P} \delta_{p, b p} \leq B P \quad C_{2}=\left\{p \mid O T P+\overline{d p} \leq t p_{p} \leq O T P+2 \overline{d p}\right\}$

$\sum_{p \in C_{k}} \sum_{b p=1}^{B P} \delta_{p, b p} \leq B P \quad C_{k}=\left\{p \mid O T P+2 \overline{d p} \leq t p_{p} \leq O T P+k \overline{d p}\right\}$

$$
\sum_{p \in D_{1}} \sum_{o=1}^{O} x_{p, o} \leq O \quad D_{1}=\left\{p \mid O T O \leq t_{p} \leq O T O+\bar{D}\right\}
$$

$$
\sum_{p \in D_{2}} \sum_{o=1}^{O} x_{p, O} \leq O \quad D_{2}=\left\{p \mid \text { OTO }+\bar{D} \leq \text { to }_{p} \leq \text { OTO }+2 \bar{D}\right\}
$$

$$
\begin{gathered}
\sum_{p \in D_{I}} \sum_{o=1}^{O} x_{p_{i} O} \leq O \quad D_{l}=\left\{p \mid \text { OTO }+\bar{D} \leq \text { to } o_{p} \leq \text { OTO }+l \bar{D}\right\} \\
d p o_{p} \geq 0 \forall p \in[1, \ldots, N] \\
x_{p_{i} o} \in\{0,1\} \forall p \in[1, \ldots, N]
\end{gathered}
$$

$$
\begin{gathered}
\delta_{p, b p} \in\{0,1\} \forall b p \in[1, \ldots, B P], \forall p \in[1, \ldots, N] \\
\alpha_{p, b m} \in\{0,1\} \forall b m \in[1, \ldots, B W m], \forall p \in[1, \ldots, N] \\
\beta_{p, b w} \in\{0,1\} \forall b w \in[1, \ldots, B W w], \forall p \in[1, \ldots, N] \\
3_{p, p} \in\{0,1\} \forall p, p \in \in[1, \ldots, N], p \neq p \\
\xi_{p, p} \in\{0,1\} \forall p, \hat{p} \in[1, \ldots, N], p \neq \hat{p} \\
v_{p, p} \in\{0,1\} \forall p, \hat{p} \in \in[1, \ldots, N], p \neq \hat{p}
\end{gathered}
$$




$$
\begin{gathered}
\pi_{p, s} \in\{0,1\} \forall s=1,2, \ldots, S, \forall p=1,2, \ldots, P \\
\lambda_{p} \in\{0,1\} \forall p \in[1, \ldots, N] \\
F_{p}^{1} \in\{0,1\} \quad \forall p=1,2, \ldots, P \\
F_{p}^{2} \in\{0,1\} \quad \forall p=1,2, \ldots, P \\
F_{p}^{3} \in\{0,1\} \quad \forall p=1,2, \ldots, P
\end{gathered}
$$

Constraints (103) to (105) check the lack of overlap between the two patients who have been allocated to an operating room at various time intervals.

\section{Computational experiments}

In this section, using the actual data associated with a first class state hospital, the model is tested and the results are presented. We assessed the whole process of rescheduling algorithm (reactive algorithm) with different scenarios.

\section{Data}

To evaluate the proposed model, a total of 52 scenarios have been tested in various sizes. This issue has been considered three operating rooms. In maximal condition, there are 7 surgeons related to 5 surgeries type, including Ophthalmic (OPT), Ear, Nose, Throat (ENT), Orthopedic (ORT), General (GEN), Obstetrics \& Gynaecology (OBG). Information about the some parameters is provided in Table 1. Based on sampling and simulations techniques, we can approximate the distribution function of the surgery duration and the other time (preparation time in operating room, cleaning time after each surgery, patient preparation time after anesthesia, patient awake time before transferring to the recovery room, etc.) to log-normal distribution function. The means and variances of these durations is given in Table 2 .

Table 1. The value of some parameters

\begin{tabular}{|c|c|c|c|}
\hline Row & Parameter & Value & Comments \\
\hline 1 & $N$ & 9 to 16 & depending on the scenario \\
\hline 2 & $P$ & $p \in\left[1_{s, n} N\right]$ & \\
\hline 3 & 0 & 3 (room) & \\
\hline 4 & $S$ & 7 (person) & \\
\hline 5 & $T_{\circ}$ & 420 (minute) & \\
\hline 6 & $T a_{\odot}$ & 180 (minute) & \\
\hline 7 & $L_{s}$ & $7(\mathrm{AM})$ & \\
\hline 8 & $U_{s}$ & $15(\mathrm{PM})$ & \\
\hline 9 & $0 \mathrm{~T}$ & 7 (AM) & \\
\hline 10 & $B W m$ & 2 (bed) & \\
\hline 11 & $B W w$ & 2 (bed) & \\
\hline 12 & $B P$ & 4 (bed) & \\
\hline
\end{tabular}




\begin{tabular}{|c|c|c|c|}
\hline 13 & $\pi_{p, s}$ & 0 or 1 & $\begin{array}{l}\text { As an example in one of the scenarios that } \mathrm{N}=10 \text {, } \\
\pi_{p, s} \text { is given in Table } 3 .\end{array}$ \\
\hline 14 & $\varepsilon_{p, 0}$ & 0 or 1 & $\begin{array}{l}\text { As an example in one of the scenarios that } \mathrm{N}=10 \text {, } \\
\qquad \varepsilon_{p, 0} \text { is given in Table } 4 .\end{array}$ \\
\hline 15 & $\lambda_{p}$ & 0 or 1 & $\begin{array}{l}\text { As an example in one of the scenarios that } \mathrm{N}=10, \\
\qquad \lambda_{p} \text { is considered as follow: } \\
\text { For } \mathrm{P}=1,2,5,8,9 \quad \lambda_{\mathrm{p}}=1_{s} \text { Others } \lambda_{\mathrm{p}}=0\end{array}$ \\
\hline 16 & $F_{p}^{a l}$ & 0 or 1 & $\begin{array}{l}\text { As an example in one of the scenarios that } \mathrm{N}=10 \text {, } \\
F_{p}^{\mathrm{a}} \text { is considered as follow: } \\
\text { For } \mathrm{P}=2,3,8,10 \quad F_{p}^{\mathrm{a}}=1 \text {, Others } F_{\mathrm{p}}^{\mathrm{a}}=0\end{array}$ \\
\hline
\end{tabular}

Table 2. The means and variances of durations (log-normal distribution)

\begin{tabular}{|c|l|c|c|}
\hline Row & Stochastic parameter & Mean & Standard deviation \\
\hline 1 & $d s_{p}$ & 30 & 10 \\
\hline 2 & $d a_{p}$ & 90 & 30 \\
\hline 3 & $d p o_{p}$ & 15 & 5 \\
\hline 4 & $d c_{p}$ & 15 & 5 \\
\hline 5 & $d p_{p}$ & 45 & 10 \\
\hline 6 & $d w_{p}$ & 20 & 5 \\
\hline
\end{tabular}

Table 3. The value of $\pi_{p, s}$ (in one of the scenarios that $N=10$ )

\begin{tabular}{|c|c|c|c|c|c|c|c|}
\hline $\boldsymbol{\pi}_{p s}$ & $\mathbf{1}$ & $\mathbf{2}$ & $\mathbf{3}$ & $\mathbf{4}$ & $\mathbf{5}$ & $\mathbf{6}$ & $\mathbf{7}$ \\
\hline $\mathbf{1}$ & 1 & 0 & 0 & 0 & 0 & 0 & 1 \\
\hline $\mathbf{2}$ & 0 & 0 & 1 & 0 & 0 & 0 & 0 \\
\hline $\mathbf{3}$ & 0 & 0 & 0 & 1 & 1 & 0 & 0 \\
\hline $\mathbf{4}$ & 0 & 1 & 0 & 0 & 0 & 0 & 0 \\
\hline $\mathbf{5}$ & 1 & 0 & 0 & 0 & 0 & 0 & 1 \\
\hline $\mathbf{6}$ & 0 & 0 & 0 & 1 & 1 & 0 & 0 \\
\hline $\mathbf{7}$ & 0 & 0 & 0 & 1 & 1 & 0 & 0 \\
\hline $\mathbf{8}$ & 1 & 0 & 0 & 0 & 0 & 0 & 1 \\
\hline $\mathbf{9}$ & 0 & 0 & 0 & 0 & 0 & 1 & 0 \\
\hline $\mathbf{1 0}$ & 1 & 0 & 0 & 0 & 0 & 0 & 1 \\
\hline
\end{tabular}

Table 4. The value of $\varepsilon_{p, o}$ (in one of the scenarios that $N=10$ )

\begin{tabular}{|c|c|c|c|}
\hline$\varepsilon_{p, 0}$ & $\mathbf{1}$ & $\mathbf{2}$ & $\mathbf{3}$ \\
\hline $\mathbf{1}$ & 1 & 1 & 1 \\
\hline $\mathbf{2}$ & 1 & 1 & 1 \\
\hline $\mathbf{3}$ & 1 & 1 & 1 \\
\hline $\mathbf{4}$ & 1 & 1 & 1 \\
\hline $\mathbf{5}$ & 1 & 1 & 1 \\
\hline $\mathbf{6}$ & 0 & 1 & 1 \\
\hline $\mathbf{7}$ & 1 & 1 & 1 \\
\hline $\mathbf{8}$ & 1 & 1 & 1 \\
\hline $\mathbf{9}$ & 1 & 0 & 0 \\
\hline $\mathbf{1 0}$ & 1 & 1 & 1 \\
\hline
\end{tabular}




\section{Results}

The models were coded in MATLAB 2014a and solved with a heuristic algorithm. We run programs on an Intel ${ }^{\circledR}$ core $^{\mathrm{TM}} \mathrm{i} 7-4500 \mathrm{U}$ CPU @ $1.80 \mathrm{GHz} 2.40 \mathrm{GHz}$ processor and 8 GB RAM. To evaluate the innovative reactive models (rescheduling algorithm), 52 samples were considered, that the number of non-electives (emergency or urgent) was between 1 to 3 and also there were 3 operating rooms. The results are presented in Table 5. On the table in front of each sample, have been provided the number of electives from basic schedule, the number of non-electives (emergency or urgent) arrived during shift, the number of electives that were rescheduled after non-electives entrance and the number of electives that were postponed to the next day.

Table 5. Results of reactive scheduling models

\begin{tabular}{|c|c|c|c|c|}
\hline $\begin{array}{l}\text { Sample } \\
\text { number }\end{array}$ & $\begin{array}{c}\text { Number of electives } \\
\text { (in basic schedule at } \\
\text { first of shift) }\end{array}$ & $\begin{array}{c}\text { Number of non-electives } \\
\text { (arrived during shift } \\
\text { and scheduled) }\end{array}$ & $\begin{array}{l}\text { Number of } \\
\text { electives } \\
\text { rescheduled }\end{array}$ & $\begin{array}{c}\text { Number of } \\
\text { electives } \\
\text { postponed }\end{array}$ \\
\hline 1 & 13 & 2 & 8 & 0 \\
\hline 2 & 10 & 1 & 5 & 0 \\
\hline 3 & 16 & 3 & 7 & 3 \\
\hline 4 & 15 & 1 & 11 & 1 \\
\hline 5 & 11 & 1 & 5 & 0 \\
\hline 6 & 14 & 1 & 7 & 1 \\
\hline 7 & 16 & 1 & 10 & 2 \\
\hline 8 & 10 & 1 & 4 & 0 \\
\hline 9 & 10 & 1 & 6 & 0 \\
\hline 10 & 16 & 1 & 8 & 3 \\
\hline 11 & 10 & 1 & 4 & 0 \\
\hline 12 & 12 & 2 & 6 & 0 \\
\hline 13 & 13 & 1 & 9 & 0 \\
\hline 14 & 12 & 2 & 4 & 0 \\
\hline 15 & 11 & 1 & 10 & 2 \\
\hline 16 & 14 & 1 & 10 & 1 \\
\hline 17 & 10 & 1 & 4 & 0 \\
\hline 18 & 15 & 1 & 6 & 1 \\
\hline 19 & 10 & 1 & 7 & 0 \\
\hline 20 & 10 & 1 & 4 & 0 \\
\hline 21 & 9 & 1 & 5 & 0 \\
\hline 22 & 16 & 2 & 12 & 4 \\
\hline 23 & 15 & 1 & 10 & 2 \\
\hline 24 & 13 & 1 & 6 & 0 \\
\hline 25 & 14 & 1 & 6 & 1 \\
\hline 26 & 13 & 1 & 7 & 0 \\
\hline 27 & 14 & 1 & 8 & 0 \\
\hline 28 & 9 & 1 & 4 & 0 \\
\hline 29 & 13 & 2 & 9 & 0 \\
\hline 30 & 10 & 1 & 5 & 0 \\
\hline 31 & 16 & 1 & 10 & 3 \\
\hline 32 & 14 & 1 & 7 & 0 \\
\hline 33 & 16 & 3 & 10 & 3 \\
\hline 34 & 9 & 1 & 4 & 0 \\
\hline 35 & 12 & 1 & 9 & 0 \\
\hline 36 & 9 & 1 & 4 & 0 \\
\hline 37 & 11 & 2 & 7 & 0 \\
\hline 38 & 10 & 1 & 6 & 0 \\
\hline 39 & 11 & 1 & 5 & 0 \\
\hline 40 & 12 & 1 & 8 & 0 \\
\hline
\end{tabular}




\begin{tabular}{|l|c|c|c|c|}
\hline 41 & 12 & 1 & 4 & 0 \\
\hline 42 & 10 & 1 & 3 & 0 \\
\hline 43 & 14 & 1 & 8 & 0 \\
\hline 44 & 15 & 2 & 11 & 2 \\
\hline 45 & 11 & 1 & 6 & 0 \\
\hline 46 & 9 & 1 & 5 & 0 \\
\hline 47 & 16 & 2 & 11 & 4 \\
\hline 48 & 14 & 1 & 9 & 0 \\
\hline 49 & 12 & 1 & 7 & 0 \\
\hline 50 & 15 & 1 & 7 & 2 \\
\hline 51 & 11 & 1 & 6 & 1 \\
\hline 52 & 9 & 1 & 2 & 0 \\
\hline
\end{tabular}

Table 6 provides patients sequence after reactive scheduling for 5 samples (for reasons of brevity, only 5 examples are presented). On the table in front of each sample, the followings have been presented: the initial sequence from basic schedule, operating rooms where non-electives (emergency or urgent) have been allocated to them (host operating room), sequence of electives and non-electives after rescheduling, postponed patients and reactive scheduling processing time.

Table 6. Patients sequence after reactive scheduling for 5 samples

\begin{tabular}{|c|c|c|c|c|c|c|}
\hline $\begin{array}{l}\text { Sample } \\
\text { number }\end{array}$ & $\begin{array}{c}\text { Operating } \\
\text { room }\end{array}$ & The initial sequence & $\begin{array}{c}\text { Host } \\
\text { operating } \\
\text { room for } \\
\text { non- } \\
\text { electives }\end{array}$ & $\begin{array}{c}\text { Sequence after } \\
\text { rescheduling }\end{array}$ & $\begin{array}{c}\text { Postponed } \\
\text { patients } \\
\text { (electives) }\end{array}$ & $\begin{array}{c}\text { Reactive } \\
\text { scheduling } \\
\text { processing } \\
\text { time (s) }\end{array}$ \\
\hline \multirow{3}{*}{1} & $o_{1}$ & $\left\{p_{2}, p_{11}, p_{5}, p_{12}\right\}$ & & $\left\{p_{1}, p_{12}\right\}$ & & \multirow{3}{*}{428} \\
\hline & $\mathrm{O}_{2}$ & $\left\{p_{4}, p_{9}, p_{1}, p_{6}, p_{2}\right\}$ & & $\left\{p_{1}, p_{2}, p_{13}\right\}$ & & \\
\hline & $o_{a}$ & $\left\{p_{10}, p_{7}, p_{12}, p_{2}\right\}$ & & $\left\{p_{6}, p \theta_{2}, p_{a}\right\}$ & & \\
\hline \multirow{3}{*}{2} & $o_{1}$ & $\left\{p_{g}, p_{g}, p_{a}, p_{1}\right\}$ & & $\left\{p_{1}, p_{6}\right\}$ & & \multirow{3}{*}{386} \\
\hline & $\mathrm{O}_{2}$ & $\left\{p_{7}, p_{10}, p_{4}\right\}$ & & $\left\{p_{10}, p_{4}\right\}$ & & \\
\hline & $o_{a}$ & $\left\{p_{5}, p_{2}, p_{6}\right\}$ & & $\left\{p e_{1}\right\}$ & & \\
\hline \multirow{3}{*}{3} & $o_{1}$ & $\left\{p_{14}, p_{11}, p_{10}, p_{12}\right\}$ & & $\left\{p \theta_{1}, p_{15}\right\}$ & \multirow{3}{*}{$p_{10} \cdot p_{12}, p_{7}$} & \multirow{3}{*}{474} \\
\hline & $o_{2}$ & $\left\{p_{6}, p_{5}, p_{2}, p_{4}, p_{15}, p_{7}\right\}$ & & $\left\{\mathrm{pe}_{2}, \mathrm{p} \theta_{2}\right\}$ & & \\
\hline & $o_{a}$ & $\left\{p_{a}, p_{1 a}, p_{9}, p_{16}, p_{d}, p_{1}\right\}$ & & $\left\{p_{1}, p_{16}, p_{4}\right\}$ & & \\
\hline \multirow{3}{*}{4} & $o_{1}$ & $\left\{p_{1}, p_{a}, p_{12}, p_{g}, p_{6}\right\}$ & & $\left\{p_{12}, p_{5}, p_{15}\right\}$ & \multirow{3}{*}{$p_{14}$} & \multirow{3}{*}{572} \\
\hline & $O_{2}$ & $\left\{p_{11}, p_{9}, p_{12}, p_{4}, p_{15}\right\}$ & & $\left\{p_{7}, p_{4}, p_{2}, p_{6}, p_{2}\right\}$ & & \\
\hline & $o_{a}$ & $\left\{p_{10}, p_{7}, p_{5}, p_{14}, p_{2}\right\}$ & & $\left\{p_{9}, p_{1 a}, p \theta_{1}\right\}$ & & \\
\hline \multirow{3}{*}{5} & $o_{1}$ & $\left\{p_{5}, p_{2}, p_{11}, p_{4}\right\}$ & & $\left\{p_{11}, p_{4}\right\}$ & & \multirow{3}{*}{381} \\
\hline & $O_{2}$ & $\left\{p_{9}, p_{1}, p_{a}\right\}$ & & $\left\{p e_{1}\right\}$ & & \\
\hline & $o_{a}$ & $\left\{p_{9}, p_{6}, p_{7}, p_{10}\right\}$ & & $\left\{p_{10}, p_{7}\right\}$ & & \\
\hline
\end{tabular}

For example, Fig. 5 is drawn for $o_{3}$ in sample 1. In the first picture the sequence of elective patients is specified $\left(\left\{p_{10}, p_{7}, p_{13}, p_{3}\right\}\right)$. When the patient $p_{7}$ is in operating room, two non-elective patients entered the hospital, in timeta. The rescheduling algorithm is used to response to non-electives and finally, reschedule process is done for all operating room. New sequence in $o_{3}$ after reschedule is presented in the second 
picture. It should be noted that after rescheduling, $p_{13}$ is scheduled in $o_{2}$. In the Fig. 5, the times are rounded.

In Table 7, the results of 52 samples in current method (manual) conditions is compared to the results of 52 samples in proposed method (reactive scheduling) conditions. The sampling in current method (manual) conditions is per number of patient (between 9 to 16) which each is repeated several times. In this table have been considered the average number of non-electives, average number of electives rescheduled, average number of electives postponed, average number of non-electives referred to other hospitals and also processing time associated with each two methods.
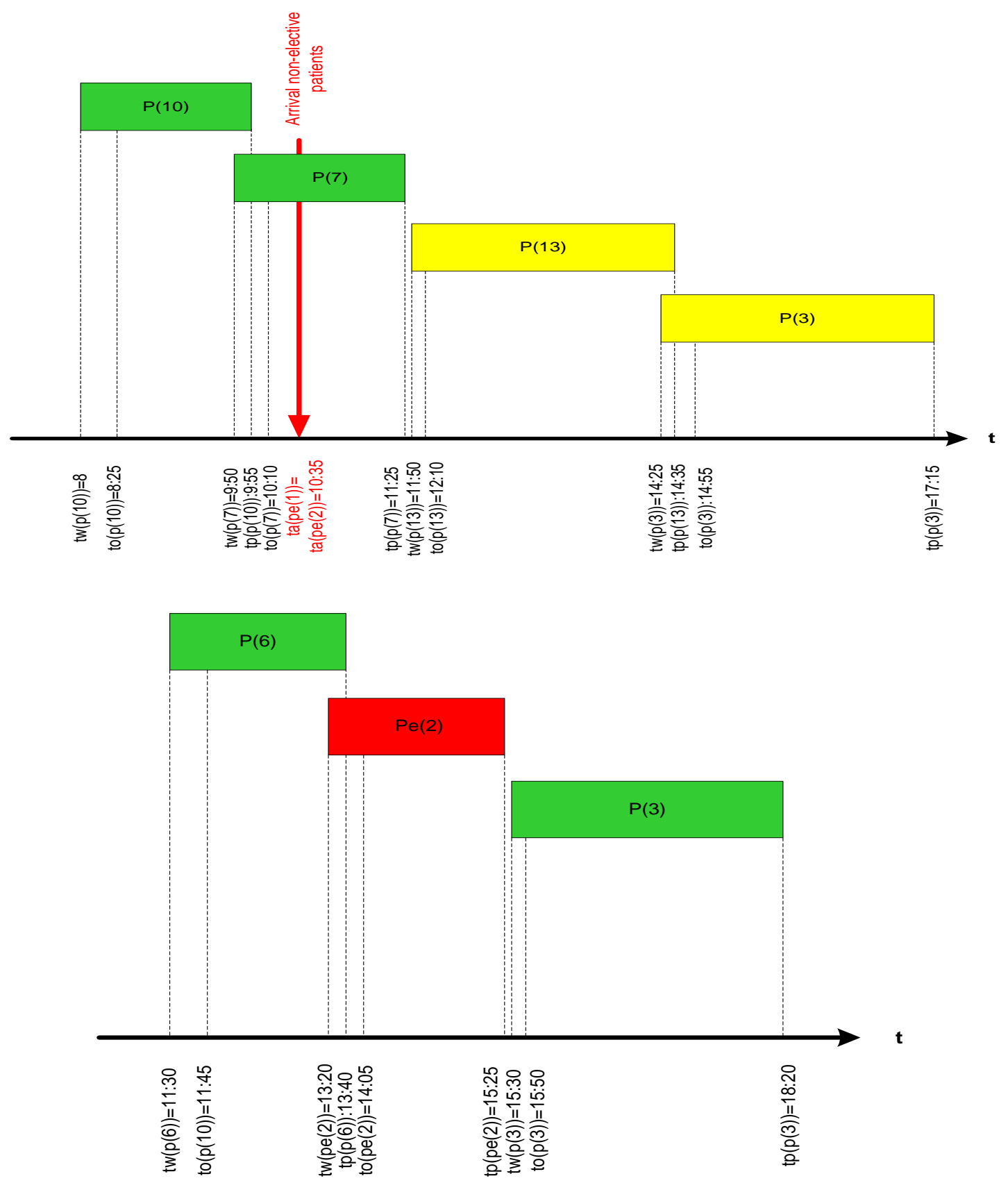

Figure 5. Sequencing of patients in operating room $o_{3}$ before and after non-electives entrance 
Table 7. Evaluations of proposed method (reactive scheduling) results compared to the current model (manual)

\begin{tabular}{|c|c|c|c|c|c|c|c|c|c|c|c|}
\hline \multirow[b]{2}{*}{ 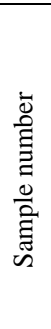 } & \multirow[b]{2}{*}{ 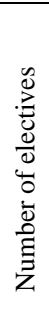 } & \multicolumn{5}{|c|}{ Current method (manual) } & \multicolumn{5}{|c|}{ Proposed method (reactive scheduling) } \\
\hline & & 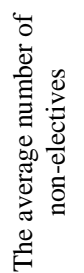 & 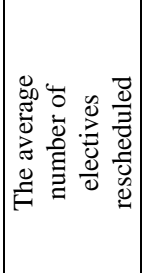 & 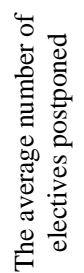 & 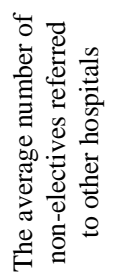 & 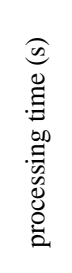 & 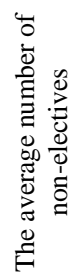 & 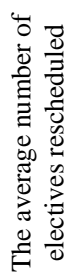 & 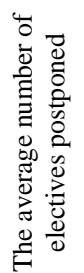 & 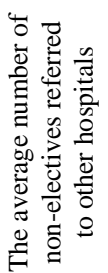 & $\begin{array}{l}0 \\
0 \\
0 \\
.0 \\
00 \\
: 0 \\
0 \\
0 \\
0 \\
0 \\
0\end{array}$ \\
\hline 1 & 9 & 1.33 & 5.16 & 0.4 & 0 & 763 & 1 & 4 & 0 & 0 & 359 \\
\hline 2 & 10 & 1 & 5.10 & 0.66 & 0 & 823 & 1 & 4.80 & 0 & 0 & 369 \\
\hline 3 & 11 & 1.10 & 6.43 & 1.38 & 0.28 & 964 & 1.17 & 6.50 & 0.50 & 0 & 393 \\
\hline 4 & 12 & 1.54 & 8 & 2.57 & 0.33 & 1468 & 1.34 & 6.33 & 0 & 0 & 432 \\
\hline 5 & 13 & 1 & 8.44 & 3.72 & 0.33 & 1419 & 1.40 & 7.80 & 0 & 0 & 437 \\
\hline 6 & 14 & 1.25 & 9.60 & 5.56 & 0.42 & 2104 & 1 & 7.86 & 0.43 & 0 & 527 \\
\hline 7 & 15 & 1.61 & 11.30 & 6.11 & 0.72 & 2537 & 1.20 & 9 & 1.60 & 0 & 583 \\
\hline 8 & 16 & 1 & 10.85 & 4.94 & 0.66 & 2443 & 1.86 & 9.71 & 3.14 & 0 & 484 \\
\hline
\end{tabular}

Comparing the results listed in Table 7 has been performed by Diagram 1, 2, 3, 4 and 5. In Diagram 1, the average number of non-electives in current and proposed method is presented. This chart indicates that the average number of non-electives in current and proposed method are almost equal (see the row 1 in Table 8).

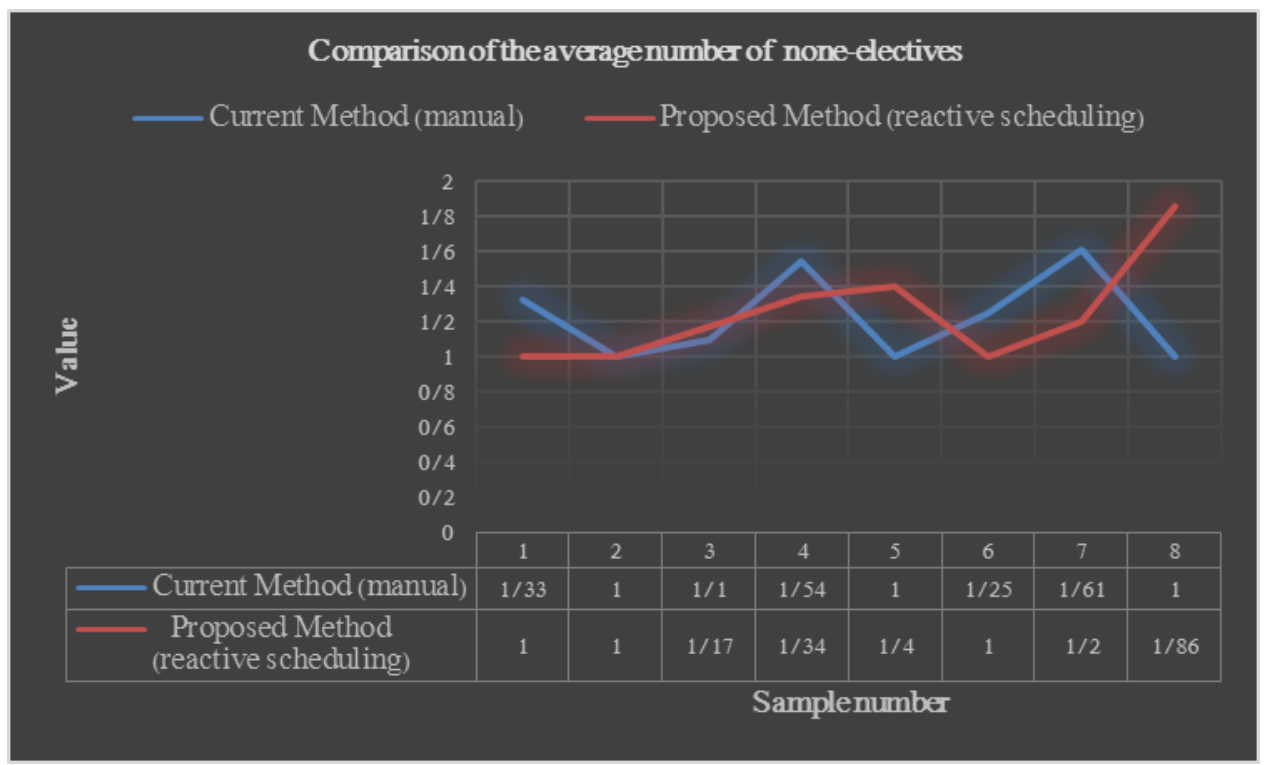

Diagram 1. Comparison of the average number of non-electives in current and proposed method

In Diagram 2, the average number of electives that were rescheduled after nonelectives entrance is presented. Fewer in number of electives rescheduled shows that the proposed model, has made less changes in the basic schedule. 


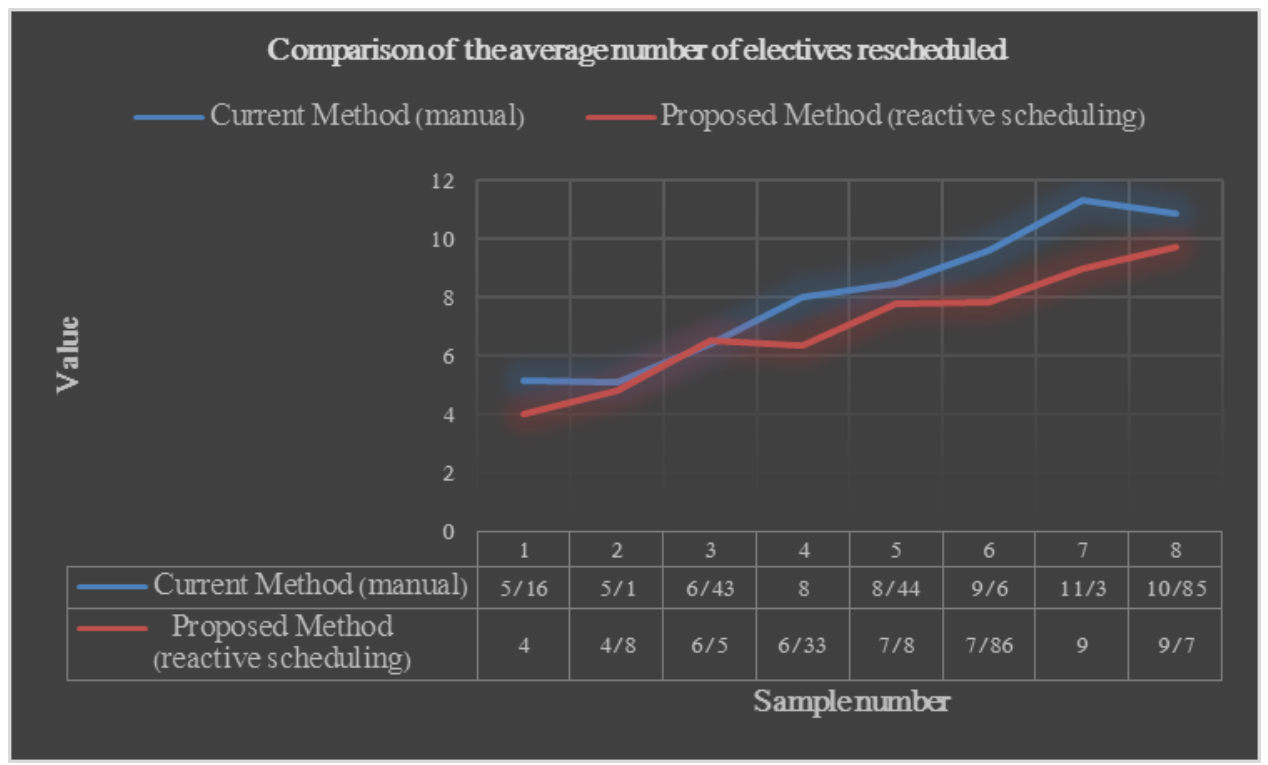

Diagram 2. Comparison of the average number of electives rescheduled in current and proposed method

In Diagram 3 the average number of electives postponed is presented. This diagram shows that the performance of the proposed model (reactive scheduling) is significantly better than the current model (manual).

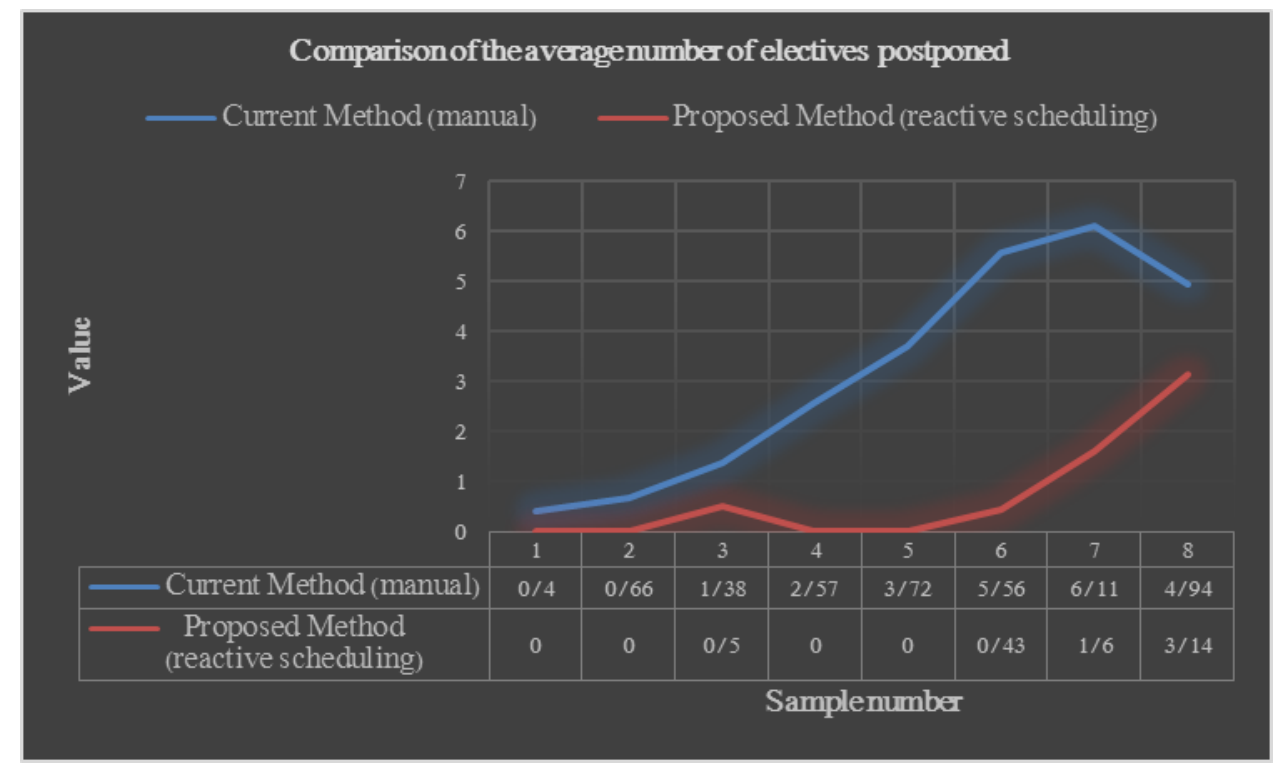

Diagram 3. Comparison of the average number of electives postponed in current and proposed method

In Diagram 4, the average number of non-electives referred to other hospitals is presented. This chart reflects the higher efficiency of the proposed model. 


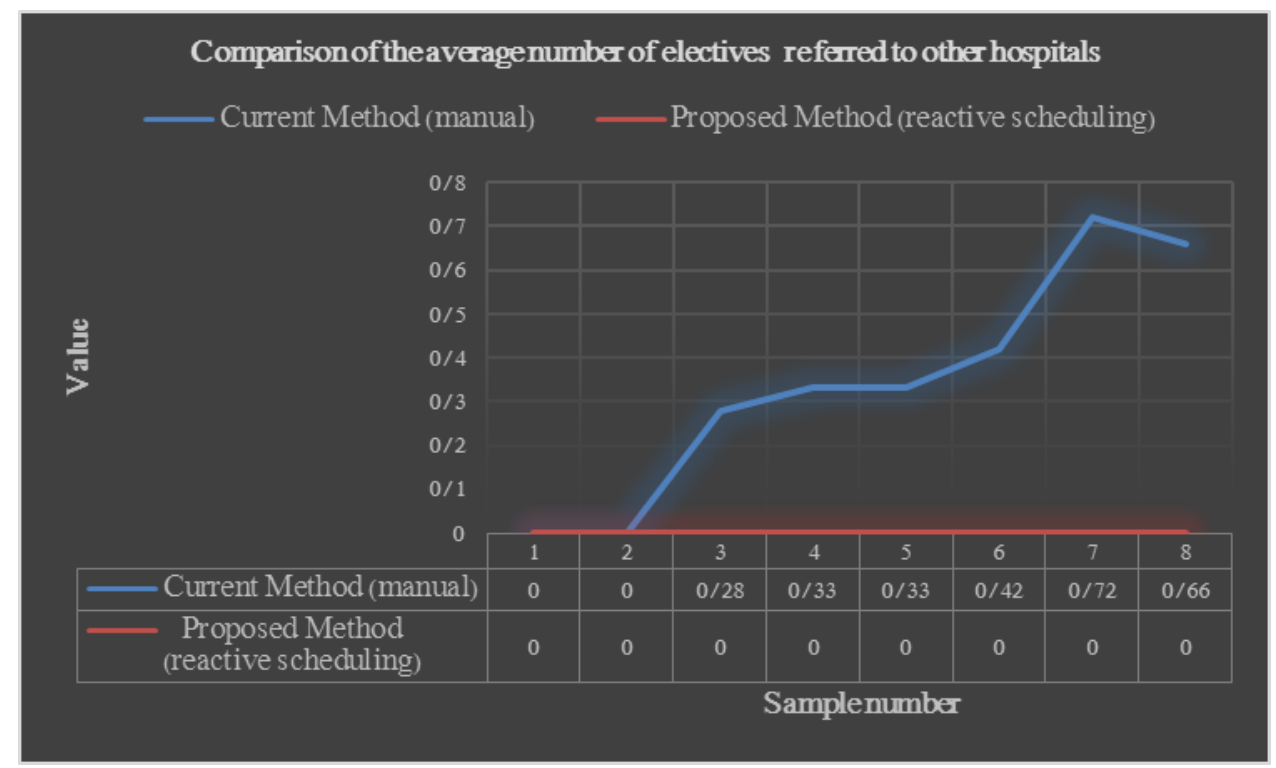

Diagram 4. Comparison of the average number of electives referred to other hospitals in current and proposed method

In Diagram 5, the processing times of two methods to reschedule patients are presented. The proposed model (reactive scheduling) is much less processing time than the current model (manual), while in the operating room time is very important and critical.

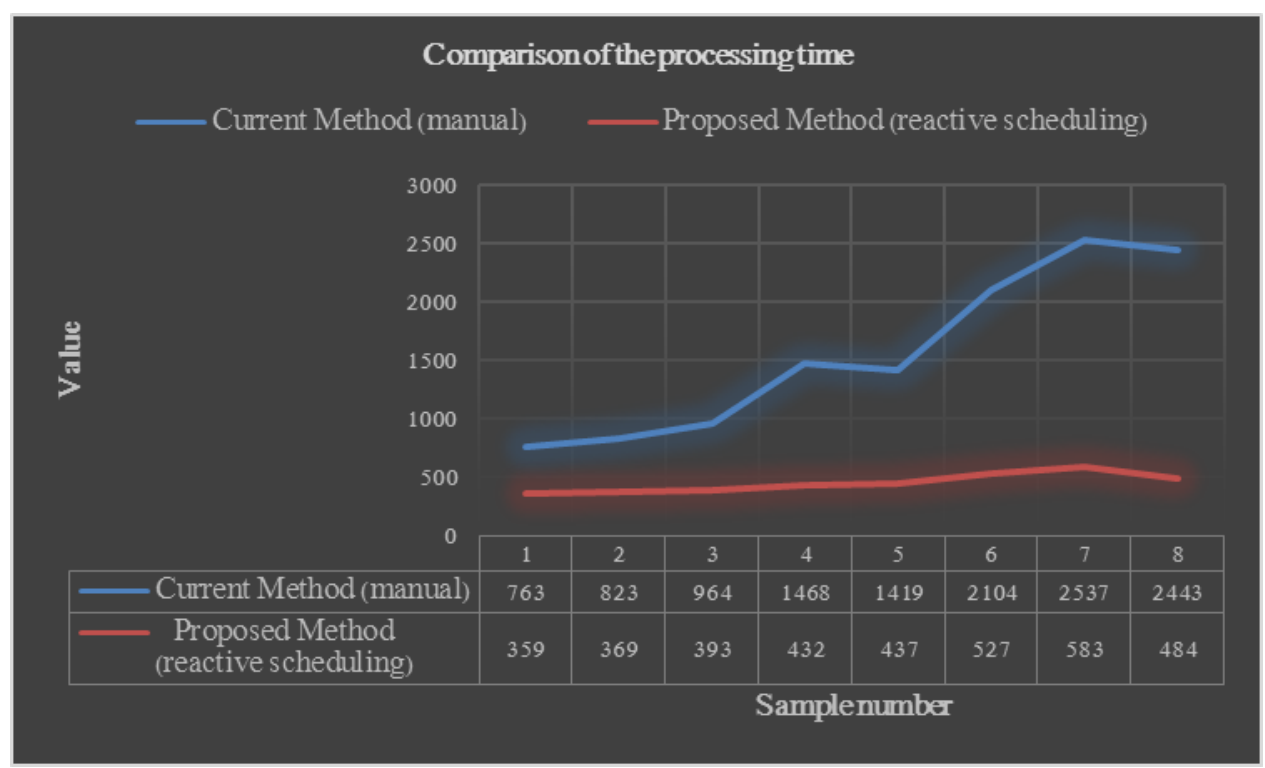

Diagram 5. Comparison of processing times in current and proposed method

Average of averages from Table 7 (average number of non-electives, average number of electives rescheduled, average number of electives postponed, average number of non-electives referred to other hospitals) is presented in Table 8. Also Table 8 shows the average of processing time in two methods. 
Table 8. Average results of the proposed method (reactive scheduling) and the current model (manual)

\begin{tabular}{ccc}
\hline & $\begin{array}{c}\text { Current method } \\
\text { (manual) }\end{array}$ & $\begin{array}{c}\text { Proposed } \\
\text { method (reactive } \\
\text { rescheduling) }\end{array}$ \\
\hline $\begin{array}{c}\text { Average of average number of non-electives } \\
\text { Average of average number of electives rescheduled } \\
\text { Average of average number of electives postponed } \\
\text { Average of average number of non-electives } \\
\text { referred to other hospitals } \\
\text { Average of processing time }\end{array}$ & 1.228 & 1.246 \\
8.11 & 7 \\
& 3.167 & 0.7087 \\
& 0.342 & 0 \\
\hline
\end{tabular}

As can be seen, the average of average of number of non-electives in current and proposed method are almost equal. Also the average of average number of electives rescheduled in reactive scheduling method compared with manual method shows about $13.6 \%$ decrease. This indicates that the proposed model, has made less changes in the basic schedule. On the other hand, the results of the proposed model present a decrease of $78 \%$ in average of average number of electives postponed. Also no non-electives has been referred to other hospitals in the reactive method. In comparing the rescheduling processing time in two methods, the reactive scheduling shows the $71 \%$ reduction.

\section{Conclusion}

In this paper, an algorithm with several stochastic mixed integer programming mathematical models are presented for the rescheduling of patients (electives and nonelectives) in operating rooms. This article has a special approach in some aspects, such as the human dimension, justice, virtue, ethics, individual freedom and rights of vulnerable social groups, including children, pregnant women, and the elderly. In mathematical schedule models, we consider three level of patient's priority $\left(\mathrm{F}_{\mathrm{p}}{ }^{1}, \mathrm{~F}_{\mathrm{p}}{ }^{2}\right.$, $\mathrm{F}_{\mathrm{p}}{ }^{3}$ ). Based on these moral and human dimensions, the decision maker can prioritize patients. In these models, the length of stay in the waiting room, the length of stay in the recovery room, surgery time, operating room preparation time, patient preparation time after anesthesia, patient awake time before being transferred to the recovery room and operating room cleaning time are stochastic. Restrictions on the parts of the before operating room (men's waiting room and women's waiting room separately) and after operating room (PACU), balance the operating rooms, priorities for patients, and also access to equipment are all from the real-world constraints, are included in this issue. In order to solve the stochastic mathematical models, a heuristic algorithm has been used. The proposed model (reactive scheduling) is evaluated and verified by solving several real examples of a first class state hospital (see Table 5). By comparing the obtained results with real data, we were able to observe the high satisfactory and excellent performance for the proposed model (see Table 7, 8). For future research, it is suggested that a model should be developed to consider the uncertainty in equipment and nonelective arrival. It is also suggested that some other optimal algorithms, such as heuristic or meta-heuristic algorithms be used for solving this problem. Furthermore, researchers can test the model in other situations such as educational hospitals or private hospitals and examine the performance of the model. 
Acknowledgments. We would like to express our sincere appreciation for the following people: Mrs. Masoumeh Abedini, Mrs. Nasrin Poursakhi, doctors and nurses of operating rooms personnel for their valuable help for providing us data for this study.

\section{REFERENCES}

[1] Abdesselam, M., Karim, A. N. M., Kays, H. M., Emrul, P., Rahman, M. A., Sarker, R. A. (2015): Formulation of an IP-based model for reactive flow-shop scheduling problem subject to arrival of new orders. - Advanced Materials Research 111(5): 616-621.

[2] Addis, B., Carello, G., Grosso, A., Lanzarone, E. (2015): Handling uncertainty in health care management using the cardinality-constrained approach: Advantages and remarks. Operations Research for Health Care 4: 1-4.

[3] Addis, B., Carello, G., Tanfani, E. (2014a): A robust optimization approach for the operating room planning problem with uncertain surgery duration. - Proceedings of the International Conference on Health Care Systems Engineering, Springer Proceedings in Mathematics \& Statistics 61: 175-189.

[4] Addis, B., Carello, G., Tanfani, E. (2014b): A robust optimization approach for the advanced scheduling problem with uncertain surgery duration in operating room planning - an extended analysis. - JIM Journal of Intelligent Manufacturing 24(3): 93-107.

[5] Atighechian, A. (2012): Scheduling of surgeries with stochastic times. - Industrial engineering department of Tarbiat-Modares University.

[6] Baumgart, A., Zoeller, A., Denz, C., Bender, H. J., Heinzl, A., Badreddin, E. (2007): Using computer simulation in operating room management: impacts on process engineering and performance. - In, 2007 Waikoloa, HI, USA. 131-137.

[7] Belien, J., Demeulemeester, E. (2007): Building cyclic master surgery schedules with leveled resulting bed occupancy. - European Journal of Operational Research 176 (2): 1185-1204.

[8] Bertsimas, D., Sim, M. (2004): The price of robustness. - Oper. Resour 52(1): 35-53.

[9] Blazewich, J., Lenstra, J. K., Rinnooykan, A. (1983): Scheduling subject to resource constraints: Classification and complexity. - Discrete Applied Mathematics 5: 11-24.

[10] Boldy, D. (1976): A review of the application of mathematical programming to tactical and strategic health and social services problems. - Operational Research Quarterly 27 (2): 439-448.

[11] Cardin, O., Trentesaux, D., Thomas, A., Castagna, P., Berger, T. (2015): Coupling predictive scheduling and reactive control in manufacturing hybrid control architectures: state of the art and future challenges. - JIM Journal of Intelligent Manufacturing 11: 1-30.

[12] Cardoen, B., Demeulemeester, E., Belien, J. (2010): Operating room planning and scheduling: A literature review. - European Journal of Operational Research 201: 921-932.

[13] Chaabane, S., Meskens, N., Guinet, A., Laurent, M. (2008): Comparison of two methods of operating theatre planning: Application in Belgian hospitals. - International Conference on Service Systems and Service Management. Troyes. 2008.

[14] Chaari, T., Chaabane, S., Aissani, N., Trentesaux, D. (2014): Scheduling under uncertainty: survey and research directions. - International Conference on Advanced logistic and Transport, 2014.

[15] Chung, S. H., Yang, M. H., Kao, C. K. (2012): Reactive scheduling to minimize makespan of parallel-machine problem with job arrival in uncertainty. - African Journal of Business Management 6(27): 7995-8009.

[16] Denton, B., Gupta, D. A. (2003): sequential bounding approach for optimal appointment scheduling. - IIE Transactions 35: 1003-1016. 
[17] Dexter, F., Macario, A., Traub, R. D., Lubarsky, D. A. (2003): Operating room utilization alone is not an accurate metric for the allocation of operating room block time to individual surgeons with low caseloads. - Anesthesiology 98: 1243-1249.

[18] Fahmy, A., Hassan, T.M., Bassioni, H. (2014): What is dynamic scheduling?. - PM World Journal 3(5): 68-79.

[19] Farrokhi, V., Kacem, I., Pokoradi, L. (2014): Ranking the solution techniques for reactive scheduling problem in operating room. - Decision and Information Technologies (CODIT), International Conference on Control, 2014.

[20] Gerami, F., Saidi-Mehrabad, M. (2014a): Allocation of patients to operating rooms under uncertainty. - International Journal of Current Life Science 4: 7121-7127.

[21] Gerami, F., Saidi-Mehrabad, M. (2014b): Allocation of patients to operating rooms under uncertainty considering the constraints after the operating room. - International Journal of Current Life Science 4: 11869-11876.

[22] Gomes, M. C., Baroso-Povoa, A. P., Novais, A. Q. (2015): Reactive scheduling in a make-to-order flexible job shop with re-entrant process and assembly: a mathematical programming approach. - International Journal of Production Research 51: 5120-5141.

[23] Hajnal, E., Almasy, G., Hunek, K. K., Kollar, G. (2007): Resource optimization by simulation technique in food logistics. - Applied Ecology and Environmental Research 5(1): 189-200.

[24] Kaur, R. (2007): Planning length of long -term field experiments through decision support systems - a case study. - Applied Ecology and Environmental Research 6(2): 63-78.

[25] Lovejoy, W. S., Li, Y. (2002): Hospital operating room capacity expansion. Management Science 48: 1369-1387.

[26] Magerlein, J., Martin, J. (1978): Surgical demand scheduling: A review. - Health Services Research 13: 418-433.

[27] Meskens, N., Duvivier, D., Hanset, A. (2013): Multi-objective operating room scheduling considering desiderata of the surgical team. - Decision Support Systems 64: 115-127.

[28] Nouaouri, I., Nicolas, J. C., Jolly, D. (2010): Reactive operating schedule in case of a disaster: arrival of unexpected victims. - In: Proceedings of the world congress on engineering 3. London, UK.

[29] Nouaouri, I., Nicolas, J. C., Jolly, D. (2011): Operating room scheduling under unexpected events: the case of a disaster. - Journal of Applied Operational Research 3(3): 163-176.

[30] Nunes, L. G. N., Decarvalho, S. V., Rodrigues, R. C. M. (2009): Markov decision process applied to the control of hospital elective admissions. - Artificial Intelligence in Medicine 47: 159-171.

[31] Persson, M. J., Persson, J. A. (2010): Analyzing management policies for operating room planning using simulation. - Health Care Management Science 13: 182-191.

[32] Saremi, A., Jula, P., Elmekkawy, T., Wang, G. (2013): Appointment scheduling of outpatient surgical services in a multistage operating room department. - Int. J. Production Economics 141: 646-658.

[33] Sciomachen, A., Tanfani, E., Testi, A. (2005): Simulation models for optimal schedules of operating theatres. - International Journal of Simulation 6: 26-34.

[34] Shamayleh, A., Fowler, G., Zhang, M. (2012): Operating room capacity planning decisions. World Academy of Science, Engineering and Technology 64: 665-669.

[35] Smith, J. C., Schaefer, A. J., Yen, J. W. (1988): A stochastic integer programming approach to solving a synchronous optical network ring design problem. - Networks 44 .

[36] Stuart, K., Kozan, E. (2012): Reactive scheduling model for the operating theatre. - Flex. Serv. Manuf. J. 24: 400-421.

[37] Testi, A., Tanfani, E., Torre, G. (2007): A three-phase approach for operating theatre schedules. - Health Care Management science 10: 163-172. 
[38] Tolner, L. (2008): The determination of parameters of multi-step adsorption isotherm by sequential simplex optimization. - Applied Ecology and Environmental Research 6(4): 111-119.

[39] Wang, B., Han, X., Zhang, X., Zhang, S. (2015): Predictive-reactive scheduling for single surgical suite subject to random emergency surgery. - Journal of Combinatorial Optimization 30(4): 949-966. 